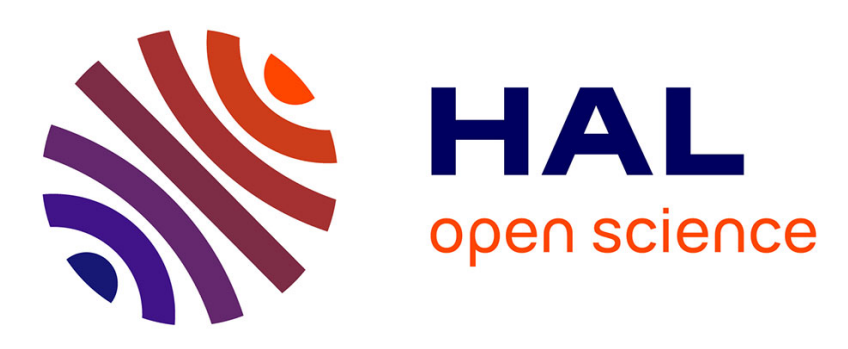

\title{
Entropy theorems in classical mechanics, general relativity, and the gravitational two-body problem
}

Marius Oltean, Luca Bonetti, Alessandro D. A. M. Spallicci, Carlos F. Sopuerta

\section{- To cite this version:}

Marius Oltean, Luca Bonetti, Alessandro D. A. M. Spallicci, Carlos F. Sopuerta. Entropy theorems in classical mechanics, general relativity, and the gravitational two-body problem. Phys.Rev.D, 2016, 94 (6), pp.064049. 10.1103/PhysRevD.94.064049 . hal-01554300

\section{HAL Id: hal-01554300 https://hal.science/hal-01554300}

Submitted on 10 Dec 2019

HAL is a multi-disciplinary open access archive for the deposit and dissemination of scientific research documents, whether they are published or not. The documents may come from teaching and research institutions in France or abroad, or from public or private research centers.
L'archive ouverte pluridisciplinaire HAL, est destinée au dépôt et à la diffusion de documents scientifiques de niveau recherche, publiés ou non, émanant des établissements d'enseignement et de recherche français ou étrangers, des laboratoires publics ou privés. 


\title{
Entropy theorems in classical mechanics, general relativity, and the gravitational two-body problem
}

\author{
Marius Oltean, ${ }^{1,2,3,4,5}$ Luca Bonetti, ${ }^{1,2,3}$ Alessandro D. A. M. Spallicci, ${ }^{1,2,3}$ and Carlos F. Sopuerta ${ }^{5}$ \\ ${ }^{1}$ Observatoire des Sciences de l'Univers en région Centre (OSUC), Université d'Orléans, \\ 1 A rue de la Férollerie, 45071 Orléans, France \\ ${ }^{2}$ Pôle de Physique, Collegium Sciences et Techniques (CoST), Université d'Orléans, \\ Rue de Chartres, 45100 Orléans, France \\ ${ }^{3}$ Laboratoire de Physique et Chimie de l'Environnement et de l'Espace (LPC2E), \\ Centre National de la Recherche Scientifique (CNRS), 3A Avenue de la Recherche Scientifique, \\ 45071 Orléans, France \\ ${ }^{4}$ Departament de Física, Facultat de Ciències, Universitat Autònoma de Barcelona, Edifici C, \\ 08193 Cerdanyola del Vallès, Spain \\ ${ }^{5}$ Institut de Ciències de l'Espai (CSIC-IEEC), Campus UAB, Carrer de Can Magrans s $/ n$, \\ 08193 Cerdanyola del Vallès, Spain
}

(Received 15 July 2016; published 19 September 2016)

\begin{abstract}
In classical Hamiltonian theories, entropy may be understood either as a statistical property of canonical systems or as a mechanical property, that is, as a monotonic function of the phase space along trajectories. In classical mechanics, there are theorems which have been proposed for proving the nonexistence of entropy in the latter sense. We explicate, clarify, and extend the proofs of these theorems to some standard matter (scalar and electromagnetic) field theories in curved spacetime, and then we show why these proofs fail in general relativity; due to properties of the gravitational Hamiltonian and phase space measures, the second law of thermodynamics holds. As a concrete application, we focus on the consequences of these results for the gravitational two-body problem, and in particular, we prove the noncompactness of the phase space of perturbed Schwarzschild-Droste spacetimes. We thus identify the lack of recurring orbits in phase space as a distinct sign of dissipation and hence entropy production.
\end{abstract}

DOI: $10.1103 /$ PhysRevD.94.064049

\section{INTRODUCTION}

The problem of reconciling the second law of thermodynamics ${ }^{1}$ with classical (deterministic) Hamiltonian evolution is among the oldest in fundamental physics [2-4]. In the context of classical mechanics (CM), this question motivated much of the development of statistical thermodynamics in the second half of the 19th century. In the context of general relativity (GR), thermodynamic ideas have occupied-and, very likely, will continue to occupya central role in our understanding of black holes and efforts to develop a theory of quantum gravity. Indeed, much work in recent years has been expended relating GR and thermodynamics [5], be it in the form of "entropic gravity" proposals [6-8] (which derive the Einstein equation from entropy formulas) or gravity-thermodynamics correspondences $[9,10]$ (wherein entropy production in GR is derived from conservation equations, in analogy with classical fluid dynamics). And yet, there is presently little consensus on the general meaning of "the entropy of a gravitational system" and still less on the question of why-purely as a consequence of the dynamical

\footnotetext{
1'It is the only physical theory of universal content concerning which I am convinced that, within the framework of applicability of its basic concepts, it will never be overthrown" [1].
}

(Hamiltonian) equations of motion-such an entropy should (strictly) monotonically increase in time, i.e. obey the second law of thermodynamics.

However one wishes to approach the issue of defining it, gravitational entropy should in some sense emerge from suitably defined (micro)states associated with the degrees of freedom not of any matter content in spacetime but of the gravitational field itself - which, in GR, means the spacetime geometry - or statistical properties thereof. Of course, we know of restricted situations in GR where we not only have entropy definitions which make sense but which also manifestly obey the second law-that is, in black hole thermodynamics. In particular, the black hole entropy is identified (up to proportionality) with its area, and hence we have that the total entropy increases when, say, two initially separated black holes merge-a process resulting, indeed, as a direct consequence of standard evolution of the equations of motion. What is noteworthy about this is that black hole entropy is thus understood not as a statistical idea but directly as a functional on the phase space of GR (comprising degrees of freedom which are subject to deterministic canonical evolution).

In $\mathrm{CM}$, the question of the statistical nature of entropy dominated many of the early debates on the origin of the second law of thermodynamics during the development of the kinetic theory of gases [2]. Initial hopes, especially by 
Boltzmann [11], were that entropy could in fact be understood as a (strictly monotonic) function on classical phase space. However, many objections soon appeared which rendered this view problematic - the two most famous being the reversibility argument of Loschmidt [12] and the recurrence theorem of Poincaré [13].

The Loschmidt reversibility argument, in essence, hinges upon the time-reversal symmetry of the canonical equations of motion and hence the ostensibly equal expectation of evolution toward or away from equilibrium. Yet, arguably, this is something which may be circumvented via a sufficiently convincing proposition for identifying the directionality of (some sort of) arrow of time - and in fact, recent work $[14,15]$ shows how this can actually be done in the Newtonian $N$-body problem, leading in this context to a clearly defined "gravitational" arrow of time. For related work in a cosmological context, see Refs. [16,17].

The Poincaré recurrence argument, on the other hand, relies on a proof that any canonical system in a bounded phase space will always return arbitrarily close to its initial state (and moreover it will do so an unbounded number of times) $[18,19]$. As the only other assumption needed for this proof is Liouville's theorem (which asserts that, in any Hamiltonian theory, the probability measure for a system to be found in an infinitesimal phase space volume is time independent), the only way for it to be potentially countered is by positing an unbounded phase space for all systems - which clearly is not the case for situations such as an ideal gas in a box.

Such objections impelled the creators of kinetic theory, Maxwell and Boltzmann in particular, to abandon the attempt to understand entropy - in what we may accordingly call a mechanical sense-as a phase space function and instead to conceive of it as a statistical notion of which the origin is epistemic ignorance, i.e. observational uncertainty of the underlying (deterministic) dynamics. The famous H-Theorem of Boltzmann [11], which was in fact initially put forth for the purposes of expounding the former, became reinterpreted and propounded in light of the latter.

Of course, later such a statistical conception of entropy came to be understood in the context of quantum mechanics via the von Neumann entropy (defined in terms of the density matrix of a quantum system) and also in the context of information theory via the Shannon entropy (defined in terms of probabilities of a generic random variable). Indeed, the meaning of the word "entropy" is now often taken to reflect an observer's knowledge (or ignorance) about the microstates of a system.

Thus, the question of why the second law of thermodynamics should hold in a Hamiltonian system may be construed within two possible formulations-on the one hand, a mechanical and, on the other, a statistical point of view. Respectively, we can state these as follows.

Problem I: Does there exist a function (or functional, if we are dealing with a field theory) on phase space which monotonically increases along the orbits of the Hamiltonian flow?
Problem II: Does there exist a function of time, defined in a suitable way in terms of a probability density on phase space, which always has a non-negative time derivative in a Hamiltonian system?

In $\mathrm{CM}$, it is Problem II that has received the most attention since the end of the 19th century. In fact, there has been significant work in recent years by mathematicians $[20,21]$ aimed at placing the statistical formulation of the $\mathrm{H}$-theorem on more rigorous footing and thus at proving more persuasively that, using appropriate assumptions, the answer to Problem II is in fact yes. In contrast, after the early Loschmidt reversibility and Poincaré recurrence arguments, Problem I has received some less well-known responses to the effect of demonstrating (even more convincingly) that the answer to it under certain conditions (to be carefully elaborated) is actually no. In this paper, we will concern ourselves with two such types of responses to Problem I: first, what we call the perturbative approach, also proposed by Poincaré [22], and, second, what we call the topological approach, due to Olsen [23] and related to the recurrence theorem. In the former, one tries to Taylor expand the time derivative of a phase space function, computed via the Poisson bracket, about a hypothetical equilibrium point in phase space, and one obtains contradictions with its strict positivity away from equilibrium. We revisit the original paper of Poincaré, clarify the assumptions of the argument, and carefully carry out the proof which is-excepting a sketch which makes it seem more trivial than it actually turns out to be-omitted therein. We furthermore extend this theorem to matter fields-in particular, a scalar and electromagnetic field-in curved spacetime. In the topological approach, on the other hand, one uses topological properties of the phase space itself to prove the nonexistence of monotonic functions. We review the proof of Olsen and discuss its connections with the recurrence theorem and more recent periodicity theorems in Hamiltonian systems from symplectic geometry.

In GR, one may consider similar lines of reasoning as in $\mathrm{CM}$ to attempt to answer Problems I and II. Naively, one might expect the same answer to Problem II, namely, yeshowever, as we will argue later in greater detail, there are nontrivial mathematical issues that need to be circumvented here even in formulating it. For Problem I, as discussed, one might confidently expect the answer to also be yes Therefore, although we do not yet know how to define entropy in GR with complete generality, we can at least ask why the proofs that furnish a negative answer to Problem I in CM fail here, and perhaps thereby gain fruitful insight into the essential features we should expect of such a definition.

Following the perturbative approach, we will show that a Taylor-expanded Poisson bracket does not contain terms which satisfy definite inequalities (as they do in $\mathrm{CM}$ ). The reason, as we will see, is that the second functional derivatives of the gravitational Hamiltonian can (unlike in $\mathrm{CM}$ ) be both positive and negative, and so its curvature 
in phase space cannot be used to constrain (functionals of) the orbits; no contradiction arises here with the second law of thermodynamics.

Following the topological approach, there are two points of view which may explicate why the proofs in CM do not carry over to GR. First, it is believed that, in general, the phase space of GR is noncompact [24]. Of course, this assertion depends on the nature of the degrees of freedom thought to be available in the spacetime under consideration, but even in very simple situations (such as cosmological spacetimes), it has been shown explicitly that the total phase space measure diverges. Physically, what this noncompactness implies is the freedom of a gravitational system to explore phase space unboundedly, without having to return (again and again) to its initial state. This leads us to the second (related) point of view as to why the topological proofs in CM fail in GR: namely, the nonrecurrence of phase space orbits. Aside from trivial situations, solutions to the canonical equations of GR are typically noncyclic (i.e. they do not close in phase space), permitting the existence of functionals which may thus increase along the Hamiltonian flow. In fact, to counter the Poincaré recurrence theorem in CM, there even exists a "no-return" theorem in GR [25-27] for spacetimes which admit compact Cauchy surfaces and satisfy suitable energy and genericity conditions; it broadly states that the spacetime cannot return, even arbitrarily close, to a previously occupied state. One might nonetheless expect nonrecurrence to be a completely general feature of all (nontrivial) gravitational systems, including spacetimes with noncompact Cauchy surfaces.

A setting of particular interest for this discussion is the gravitational two-body problem. With the recent detection [28] (and ongoing efforts toward further observations [29]) of gravitational waves from two-body systems, the emission of which ought to be closely related to entropy production, a precise understanding and quantification of the latter is becoming more and more salient. In the CM two-body (i.e. Kepler) problem, the consideration of Problem I clearly explains the lack of entropy production due to phase space compactness (for a given finite range of initial conditions). In the Newtonian $N$-body problem, where (as we will elaborate) neither the perturbative nor the topological proofs are applicable, the answer to Problem I was actually shown to be yes in Refs. $[14,15]$. In GR, the two-body problem may be considered in the context of perturbed Schwarzschild-Droste (SD) spacetimes ${ }^{2}$ (as is relevant, for instance, in the context of extreme-mass-ratio inspirals). Here, the phase space volume (symplectic) form has been explicitly computed in Ref. [34]. We will use this in this paper to show that in such spacetimes

\footnotetext{
${ }^{2}$ Commonly, this is referred to simply as the "Schwarzschild metric." Yet, it has long gone unrecognized that Johannes Droste, then a doctoral student of Lorentz, discovered this metric independently and announced it only four months after Schwarzschild [30-33], so for the sake of historical fairness, we here use the nomenclature "Schwarzschild-Droste metric" instead.
}

the phase space is noncompact; hence, there are no contradictions with nonrecurrence or entropy production.

We structure this paper as follows. In Sec. II, we establish some basic notation for describing general (constrained) Hamiltonian systems. In Secs. III and IV, we address Problem I via the approaches described in this Introduction in CM and GR, respectively. Then, in Sec. V, we apply our discussion to the gravitational two-body problem, and finally in Sec. VI, we conclude.

\section{SETUP}

We begin by establishing some basic notation for describing general canonical theories which will be pertinent for our discussion. Technical details and definitions are relegated to Appendix A, and comprehensive expositions can be found in Refs. [35,36].

Let $\mathscr{Q}$ denote the space of admissible configurations for any given classical system (of particles and/or fields), the dynamics of which can be determined from a given Lagrangian function $L$. From this, one may cast the theory in canonical form; its degrees of freedom are then represented by a phase space $\mathscr{P}$ (which is the cotangent bundle of $\mathscr{Q}$ ), and its dynamics are determined by a Hamiltonian function $H: \mathscr{P} \rightarrow \mathbb{R}$. In the case of field theories, the term phase space "function" should be understood as "functional" (of the fields).

The phase space $\mathscr{P}$ is, by construction, a symplectic manifold. Let $\boldsymbol{\omega}$ denote the symplectic form on $\mathscr{P}$ and $\Omega$ the volume form obtained therefrom. (The latter, when integrated over $\mathscr{P}$, gives its total volume or measure, $\mu(\mathscr{P})=\int_{\mathscr{P}} \Omega$.) Furthermore, let $\boldsymbol{X}_{F}$ be the Hamiltonian vector field of any phase space function $F: \mathscr{P} \rightarrow \mathbb{R}$. The time evolution of the system through $\mathscr{P}$ is represented by the integral curves of this vector field for the Hamiltonian function, $\boldsymbol{X}_{H}$. We denote the Hamiltonian flow (i.e. the flow generated by $\boldsymbol{X}_{H}$ ), for some time interval $\mathscr{T} \subseteq \mathbb{R}$, by $\Phi_{t}: \mathscr{P} \times \mathscr{T} \rightarrow \mathscr{P}$.

The situation becomes more subtle if the system under consideration is constrained (as is the case, for example, with Maxwellian electromagnetism (EM) or GR). Physically, the existence of constraints in a theory means that not all points in $\mathscr{P}$ are dynamically accessible; in general, not all initial conditions are permissible, and not all points in $\mathscr{P}$ can be reached from permissible initial conditions. The consequence is that one can no longer use the phase space $\mathscr{P}$ but must instead work with a reduced phase space $\mathscr{S}$, the symplectic form of which we denote by $\left.\boldsymbol{\omega}\right|_{\mathscr{S}}$. The details of how this must be constructed are offered in Appendix A.

In what follows, what will be important is the reduced phase space measure $\mu(\mathscr{S})$. It can be computed by integrating the volume form $\left.\Omega\right|_{\mathscr{S}}$ of $\mathscr{S}$ (determined by $\left.\omega\right|_{\mathscr{S}}$ ),

$$
\mu(\mathscr{S})=\left.\int_{\mathscr{S}} \Omega\right|_{\mathscr{S}}
$$


We will see that the topological approach toward the validity of the second law of thermodynamics (described in the Introduction) relies crucially on whether or not this quantity is divergent.

In situations where the implication is clear, we may drop the term "reduced" when making statements about the reduced phase space. Finally, using all this, we can now restate more precisely the above two problems on the second law of thermodynamics.

Problem I (restatement): Does there exist any $S: \mathscr{S} \rightarrow \mathbb{R}$ that monotonically increases along the orbits of $\Phi_{t}$ ?

Problem II (restatement): Does there exist any $S: \mathscr{T} \rightarrow \mathbb{R}$, defined in a suitable way in terms of a probability density $\rho: \mathscr{S} \times \mathscr{T} \rightarrow[0,1]$, satisfying $\mathrm{d} S / \mathrm{d} t \geq$ 0 in a Hamiltonian system? [Traditionally, the definition taken here for entropy is (a coarse-grained version of) $S(t)=-\int_{\mathscr{P}} \Omega \rho \ln \rho$, or its appropriate reduction to $\mathscr{S}$ if there are constraints.]

\section{ENTROPY THEOREMS IN CLASSICAL MECHANICS}

\section{A. Setup}

Classical particle mechanics with $N$ degrees of freedom [18] can be formulated as a Lagrangian theory with an $N$-dimensional configuration space $\mathscr{Q}$. This means that we will have a canonical theory on a $2 N$-dimensional phase space $\mathscr{P}$. We can choose canonical coordinates $\left(q_{1}, \ldots, q_{N}\right)$ with conjugate momenta $\left(p_{1}, \ldots, p_{N}\right)$ such that the symplectic form on $\mathscr{P}$ is given by

$$
\boldsymbol{\omega}=\sum_{j=1}^{N} \mathrm{~d} p_{j} \wedge \mathrm{d} q_{j} .
$$

Then, the volume form on $\mathscr{P}$ is simply the $N$ th exterior power of the symplectic form, in particular $\Omega=\left[(-1)^{N(N-1) / 2} / N !\right] \boldsymbol{\omega}^{\wedge N}$, and $\boldsymbol{X}_{H}$ is here given in coordinates by

$$
\boldsymbol{X}_{H}=\sum_{j=1}^{N}\left(\frac{\partial H}{\partial p_{j}} \frac{\partial}{\partial q_{j}}-\frac{\partial H}{\partial q_{j}} \frac{\partial}{\partial p_{j}}\right) .
$$

The action of $\boldsymbol{X}_{H}$ on any phase space function $F: \mathscr{P} \rightarrow \mathbb{R}$, called the Poisson bracket, gives its time derivative:

$$
\dot{F}=\frac{\mathrm{d} F}{\mathrm{~d} t}=\boldsymbol{X}_{H}(F)=\{F, H\} .
$$

We obtain from this $\dot{q}_{j}=\left\{q_{j}, H\right\}=\partial H / \partial p_{j}$ and $\dot{p}_{j}=\left\{p_{j}, H\right\}=-\partial H / \partial q_{j}$, which are the canonical equations of motion. Moreover, we have that the symplectic form of $\mathscr{P}$, and hence its volume form, are preserved along $\Phi_{t}$; in other words, we have $\mathcal{L}_{\boldsymbol{X}_{H}} \boldsymbol{\omega}=0=\mathcal{L}_{\boldsymbol{X}_{H}} \Omega$, which is known as Liouville's theorem.
We now turn to address Problem I in CM-that is, the question of whether there exists a function $S: \mathscr{P} \rightarrow \mathbb{R}$ that behaves like entropy in a classical Hamiltonian system. Possibly the most well-known answer given to this is the Poincaré recurrence theorem, the proof of which we present in Appendix A.

Let us now discuss, in turn, the perturbative and topological approaches.

\section{B. Perturbative approach}

We revisit and carefully explicate, in this subsection, the argument given by Poincare [22] to the effect that an entropy function $S: \mathscr{P} \rightarrow \mathbb{R}$ does not exist. First, we will clarify the assumptions that need to go into it, i.e. the conditions we must impose both on the entropy $S$ as well as on the Hamiltonian $H$, and then we will supply a rigorous proof.

\section{Review of Poincaré's idea for a proof}

In his original paper [22] (translated into English in Ref. [23]), the argument given by Poincaré (expressed using the contemporary notation of this paper) for the nonexistence of such a function $S: \mathscr{P} \rightarrow \mathbb{R}$ is the following: if $S$ behaves indeed like entropy, it should satisfy

$$
\dot{S}=\{S, H\}=\sum_{k=1}^{N}\left(\frac{\partial H}{\partial p_{k}} \frac{\partial S}{\partial q_{k}}-\frac{\partial H}{\partial q_{k}} \frac{\partial S}{\partial p_{k}}\right)>0
$$

around a hypothetical equilibrium point in $\mathscr{P}$. Taylor expanding each term and assuming all first partials of $S$ and $H$ vanish at this equilibrium, we obtain a quadratic form (in the distances away from equilibrium) plus higher-order terms. If we are "sufficiently close" to equilibrium, we may ignore the higher-order terms and simply consider the quadratic form, which thus needs to be positive definite for the above inequality [Eq. (5)] to hold. But here, Poincaré, without presenting any further explicit computations, simply asserts that "it is easy to satisfy oneself that this is impossible if one or the other of the two quadratic forms $S$ and $H$ is definite, which is the case here." (Our modern language modification is in italic.)

Neither the casual dismissal of the higher-order terms nor, even more crucially, the fact that "it is easy to satisfy oneself" of the impossibility of this quadratic form to be positive definite is immediately apparent from this discussion. In fact, all of the points in this line of reasoning require a careful statement of the necessary assumptions as well as some rather nontrivial details of the argumentation required to obtain the conclusion (that $\dot{S}=0$ ).

In what follows, we undertake precisely that. First, we look at the assumptions needed for this method to yield a useful proof, and then we carry out the proof in full detail and rigor. 


\section{Entropy conditions}

A function $S: \mathscr{P} \rightarrow \mathbb{R}$ can be said to behave like entropy insofar as it satisfies the laws of thermodynamics. In particular, it should conform to two assumptions: first, that it should have an equilibrium point and, second, that it should obey the second law of thermodynamics-which heuristically states that it should be increasing in time everywhere except at the equilibrium point, where it should cease to change. We state these explicitly as follows:

S1 (existence of equilibrium): We assume there exists a point in phase space, $x_{0} \in \mathscr{P}$, henceforth referred to as the "equilibrium" configuration of the system, which is a stationary point of the entropy $S$, i.e. all first partials thereof should vanish when evaluated there,

$$
\left(\frac{\partial S}{\partial q_{j}}\right)_{0}=0=\left(\frac{\partial S}{\partial p_{j}}\right)_{0}
$$

where, for convenience, we use the notation $(\cdot)_{0}=\left.(\cdot)\right|_{x_{0}}$ to indicate quantities evaluated at equilibrium. Note that by the definition of the Poisson bracket [Eq. (4)], this implies $(\dot{S})_{0}=0$.

S2 (second law of thermodynamics): A common formulation of the second law asserts that the entropy $S$ is always increasing in time when the system is away from equilibrium (i.e. $\dot{S}>0$ everywhere in $\mathscr{P} \backslash x_{0}$ ) and attains its maximum value at equilibrium, where it ceases to change in time (i.e. $\dot{S}=0$ at $x_{0}$, as implied by the first condition). We need to work, however, with a slightly stronger version of the second law: namely, the requirement that the Hessian matrix of $\dot{S}$,

$$
\operatorname{Hess}(\dot{S})=\left[\begin{array}{c|c}
\frac{\partial^{2} \dot{S}}{\partial q_{i} \partial q_{j}} & \frac{\partial^{2} \dot{S}}{\partial q_{i} \partial p_{j}} \\
\hline \frac{\partial^{2} \dot{S}}{\partial p_{i} \partial q_{j}} & \frac{\partial^{2} \dot{S}}{\partial p_{i} \partial p_{j}}
\end{array}\right],
$$

is positive definite when evaluated at equilibrium, i.e. $(\operatorname{Hess}(\dot{S}))_{0} \succ 0$.

We make now a few remarks about these assumptions. First, S2 is a sufficient-though not strictly necessarycondition to guarantee $\dot{S}>0$ in $\mathscr{P} \backslash x_{0}$ and $\dot{S}=0$ at $x_{0}$. However, the assumption of positive definiteness of the Hessian of the entropy $S$ itself at equilibrium is often used in statistical mechanics [37], and so it may not be objectionable to extend this supposition to $\dot{S}$ as well. (In any case, this leaves out only special situations where higher-order derivative tests are needed to certify the global minimization of $\dot{S}$ at equilibrium, which arguably are more of mathematical rather than physical interest; we may reasonably expect the entropy as well as its time derivative to be quadratic in the phase space variables as a consequence of its ordinary statistical mechanics definitions in terms of energy.)

Second, the above two conditions omit the consideration of functions on $\mathscr{P}$ which are everywhere strictly monotonically increasing in time, i.e. the time derivative of which is always positive with no equilibrium point. The topological approaches to Problem I, which we will turn to in the next subsection, do accommodate the possibility of such functions.

Third, the equilibrium point $x_{0} \in \mathscr{P}$, though usually (physically) expected to be unique, need not be for the purposes of what follows, so long as it obeys the two conditions S1 and S2. In other words, it suffices that there exists at least one such point in $\mathscr{P}$.

Fourth, there is no topological requirement being imposed on the phase space $\mathscr{P}$. It is possible, in other words, for its total measure $\mu(\mathscr{P})=\int_{\mathscr{P}} \Omega$ to diverge. This means that the theorem applies to systems which can, in principle, explore phase space unboundedly, without any limits being imposed (either physically or mathematically) thereon.

\section{Hamiltonian conditions}

Next, we make a few assumptions about the Hamiltonian $H: \mathscr{P} \rightarrow \mathbb{R}$ which we need to impose in order to carry out our proof. The first two assumptions are reasonable for any typical Hamiltonian in classical mechanics, as we will discuss. The third, however, is stronger than necessary to account for all Hamiltonians in general—and indeed, as we will see, unfortunately leaves out certain classes of Hamiltonians of interest. However, we regard it as a necessary assumption which we cannot relax in order to formulate the proof according to this approach. Our assumptions on $H$ are thus as follows:

$H 1$ (kinetic terms): With regard to the second partials of $H$ with respect to the momentum variables, we assume the following:

(a) We can make a choice of coordinates so as to diagonalize (i.e. decouple) the kinetic terms. In other words, we can choose to write $H$ in such a form that we have:

$$
\frac{\partial^{2} H}{\partial p_{i} \partial p_{j}}=\delta_{i j} \frac{\partial^{2} H}{\partial p_{j}^{2}}
$$

(b) Additionally, the second partials of $H$ with respect to each momentum variable, representing the coefficients of the kinetic terms, should be non-negative:

$$
\frac{\partial^{2} H}{\partial p_{j}^{2}} \geq 0
$$

$H 2$ (mixed terms): We assume that we can decouple the terms that mix kinetic and coordinate degrees of freedom (via performing integrations by parts, if necessary, in the action out of which the Hamiltonian is constructed), such that $H$ can be written in a form where 


$$
\frac{\partial^{2} H}{\partial p_{i} \partial q_{j}}=0 .
$$

H3 (potential terms): We need to restrict our consideration to Hamiltonians of which the partial Hessian with respect to the coordinate variables is positive semidefinite at the point of equilibrium (assuming it exists), i.e. $\left[\partial^{2} H / \partial q_{i} \partial q_{j}\right]_{0} \geq 0$. In fact, we need to impose a slightly stronger (sufficient, though not strictly necessary) condition: that any of the row sums of $\left[\partial^{2} H / \partial q_{i} \partial q_{j}\right]_{0}$ are non-negative. That is to say, we assume

$$
\sum_{i=1}^{N}\left(\frac{\partial^{2} H}{\partial q_{i} \partial q_{j}}\right)_{0} \geq 0
$$

We can make a few remarks about these assumptions. First, $\mathrm{H} 1$ and $\mathrm{H} 2$ are manifestly satisfied for the most typically encountered form of the Hamiltonian in CM,

$$
H=\sum_{j=1}^{N} \frac{p_{j}^{2}}{2 m_{j}}+V\left(q_{1}, \ldots, q_{N}\right),
$$

where $m_{j}$ are the masses associated with each degree of freedom and $V$ is the potential (a function of only the configuration variables, and not the momenta). Indeed, $\mathrm{H} 1$ (a) is satisfied since we have $\partial^{2} H / \partial p_{i} \partial p_{j}=0$ unless $i=j$, regardless of $V$. For H1(b), we clearly have $\partial^{2} H / \partial p_{j}^{2}=1 / m_{j}>0$ assuming masses are positive. (Theories with negative kinetic terms, i.e. "ghosts," are ordinarily thought of as being problematic.) Finally, $\mathrm{H} 2$ holds as $\partial^{2} H / \partial p_{i} \partial q_{j}=0$ is satisfied by construction.

Second, For typical Hamiltonians [Eq. (12)], H3 translates into a condition on the potential $V$, i.e. the requirement that $\sum_{i=1}^{N}\left(\partial^{2} V / \partial q_{i} \partial q_{j}\right)_{0} \geq 0$. This is not necessarily satisfied in general in CM, though it is for many systems. For example, when we have just 1 degree of freedom, $N=1$, this simply means that the potential $V(q)$ is concave upward at the point of equilibrium (thus regarded as a stable equilibrium), i.e. $\left(\mathrm{d}^{2} V(q) / \mathrm{d} q^{2}\right)_{0} \geq 0$, which is reasonable to assume. As another example, for a system of harmonic oscillators with no interactions, $V=$ $(1 / 2) \sum_{j=1}^{N} m_{j} \omega_{j}^{2} q_{j}^{2}$, we clearly have $\sum_{i=1}^{N} \partial^{2} V / \partial q_{i} \partial q_{j}=$ $m_{j} \omega_{j}^{2}>0$ for positive masses. Indeed, even introducing interactions does not change this so long as the couplings are mostly non-negative. (In other words, if the negative couplings do not dominate in strength over the positive ones.) Higher (positive) powers of the $q_{j}$ variables in $V$ are also admissible under a similar argument. However, we can see that condition H3 [Eq. (11)] excludes certain classes of inverse-power potentials. Most notably, it excludes the Kepler (gravitational two-body) Hamiltonian,
$H=(1 / 2 m)\left(p_{1}^{2}+p_{2}^{2}\right)-G M m /\left(q_{1}^{2}+q_{2}^{2}\right)^{1 / 2}$, where $q_{j}$ are the Cartesian coordinates in the orbital plane and $p_{j}$ are the associated momenta. In this case, we have $\operatorname{det}\left(\left[\partial^{2} H / \partial q_{i} \partial q_{j}\right]\right)=-2(G M m)^{2} /\left(q_{1}^{2}+q_{2}^{2}\right)^{3}<0$, and hence $\left[\partial^{2} H / \partial q_{i} \partial q_{j}\right]$ is negative definite everywhere and therefore cannot satisfy H3 [Eq. (11)].

\section{Our proof}

We will now show that there cannot exist a function $S: \mathscr{P} \rightarrow \mathbb{R}$ satisfying the assumptions $\mathrm{S} 1$ and $\mathrm{S} 2$ of Sec. III B 2 in a Hamiltonian system that obeys the assumptions $\mathrm{H} 1-\mathrm{H} 3$ of Sec. III B 3 on $H: \mathscr{P} \rightarrow \mathbb{R}$. We do this by simply assuming that such a function exists, and we will show that this implies a contradiction. For a pictorial representation, see Fig. 1.

$N=1$ : Let us first carry out the proof for $N=1$ degree of freedom so as to make the argument for general $N$ easier to follow. Let $S: \mathscr{P} \rightarrow \mathbb{R}$ be any function on the configuration space $\mathscr{P}$ satisfying assumptions $\mathrm{S} 1$ and $\mathrm{S} 2$ of Sec. III B 2; i.e. it has an equilibrium point, and the Hessian of its time derivative is positive definite there. We know that its time derivative at any point $x=(q, p) \in$ $\mathscr{P}$ can be evaluated, as discussed in Sec. III A, via the Poisson bracket:

$$
\dot{S}=\frac{\partial H}{\partial p} \frac{\partial S}{\partial q}-\frac{\partial H}{\partial q} \frac{\partial S}{\partial p} .
$$

Let us now insert into this the Taylor series for each term expanded about the equilibrium point $x_{0}=\left(q_{0}, p_{0}\right)$. Denoting $\Delta q=q-q_{0}$ and $\Delta p=p-p_{0}$, and using $\mathcal{O}\left(\Delta^{n}\right)$ to represent $n$th order terms in products of $\Delta q$ and $\Delta p$, we have

$\frac{\partial H}{\partial q}=\left(\frac{\partial H}{\partial q}\right)_{0}+\left(\frac{\partial^{2} H}{\partial q^{2}}\right)_{0} \Delta q+\left(\frac{\partial^{2} H}{\partial p \partial q}\right)_{0} \Delta p+\mathcal{O}\left(\Delta^{2}\right)$

and similarly for the $p$ partial of $H$, while

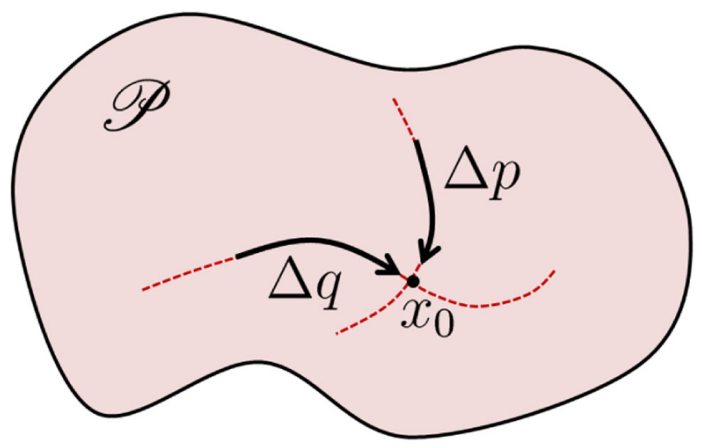

FIG. 1. The idea of the perturbative approach is to evaluate $\dot{S}$ along different directions in phase space away from equilibrium and arrive at a contradiction with its strict positivity. 


$$
\frac{\partial S}{\partial q}=\left(\frac{\partial^{2} S}{\partial q^{2}}\right)_{0} \Delta q+\left(\frac{\partial^{2} S}{\partial p \partial q}\right)_{0} \Delta p+\mathcal{O}\left(\Delta^{2}\right)
$$

and similarly for the $p$ partial of $S$, where we have used the condition S1 [Eq. (6)] which entails that the zero-order term vanishes. Inserting all Taylor series into the Poisson bracket [Eq. (13)] and collecting terms, we obtain the following result,

$\dot{S}=\left[\begin{array}{ll}a & b\end{array}\right]\left[\begin{array}{l}\Delta q \\ \Delta p\end{array}\right]+\left[\begin{array}{ll}\Delta q & \Delta p\end{array}\right]\left[\begin{array}{ll}A & B \\ B & C\end{array}\right]\left[\begin{array}{l}\Delta q \\ \Delta p\end{array}\right]+\mathcal{O}\left(\Delta^{3}\right)$,

where,

$$
\begin{aligned}
& a=\left(\frac{\partial H}{\partial p}\right)_{0}\left(\frac{\partial^{2} S}{\partial q^{2}}\right)_{0}-\left(\frac{\partial H}{\partial q}\right)_{0}\left(\frac{\partial^{2} S}{\partial q \partial p}\right)_{0}, \\
& b=\left(\frac{\partial H}{\partial p}\right)_{0}\left(\frac{\partial^{2} S}{\partial p \partial q}\right)_{0}-\left(\frac{\partial H}{\partial q}\right)_{0}\left(\frac{\partial^{2} S}{\partial p^{2}}\right)_{0},
\end{aligned}
$$

and

$$
\begin{aligned}
& A=\left(\frac{\partial^{2} H}{\partial q \partial p}\right)_{0}\left(\frac{\partial^{2} S}{\partial q^{2}}\right)_{0}-\left(\frac{\partial^{2} H}{\partial q^{2}}\right)_{0}\left(\frac{\partial^{2} S}{\partial q \partial p}\right)_{0}, \\
& B=\frac{1}{2}\left[\left(\frac{\partial^{2} H}{\partial p^{2}}\right)_{0}\left(\frac{\partial^{2} S}{\partial q^{2}}\right)_{0}-\left(\frac{\partial^{2} H}{\partial q^{2}}\right)_{0}\left(\frac{\partial^{2} S}{\partial p^{2}}\right)_{0}\right], \\
& C=\left(\frac{\partial^{2} H}{\partial p^{2}}\right)_{0}\left(\frac{\partial^{2} S}{\partial p \partial q}\right)_{0}-\left(\frac{\partial^{2} H}{\partial p \partial q}\right)_{0}\left(\frac{\partial^{2} S}{\partial p^{2}}\right)_{0} .
\end{aligned}
$$

By assumption $\mathrm{S} 2$, we have that $\dot{S}$ as given above [Eq. (16)] is strictly positive for any $x \neq x_{0}$ in $\mathscr{P}$. In particular, let $\delta>0$, and let us consider $\dot{S}$ [Eq. (16)] evaluated at the sequence of points $\left\{x_{n}^{ \pm}\right\}_{n=1}^{\infty}$, where $x_{n}^{ \pm}=\left(q_{0} \pm \delta / n, p_{0}\right)$, such that the only deviation away from equilibrium is along the direction $\Delta q= \pm \delta / n$, with all other $\Delta p$ vanishing. Then, for any $n$, we must have according to our expression for $\dot{S}$ [Eq. (16)]

$$
\begin{gathered}
\dot{S}\left(x_{n}^{+}\right)=a \frac{\delta}{n}+A \frac{\delta^{2}}{n^{2}}+\mathcal{O}\left(\frac{\delta^{3}}{n^{3}}\right)>0, \\
\dot{S}\left(x_{n}^{-}\right)=-a \frac{\delta}{n}+A \frac{\delta^{2}}{n^{2}}+\mathcal{O}\left(\frac{\delta^{3}}{n^{3}}\right)>0 .
\end{gathered}
$$

Taking the $n \rightarrow \infty$ limit of the first inequality implies $a \geq 0$, while doing the same for the second inequality implies $a \leq 0$. Hence, $a=0$. A similar argument (using $\Delta p= \pm \delta / n$ ) implies $b=0$. Thus, $S$ needs to satisfy the constraints

$$
0=\left(\frac{\partial H}{\partial p}\right)_{0}\left(\frac{\partial^{2} S}{\partial q^{2}}\right)_{0}-\left(\frac{\partial H}{\partial q}\right)_{0}\left(\frac{\partial^{2} S}{\partial q \partial p}\right)_{0}
$$

$$
0=\left(\frac{\partial H}{\partial p}\right)_{0}\left(\frac{\partial^{2} S}{\partial p \partial q}\right)_{0}-\left(\frac{\partial H}{\partial q}\right)_{0}\left(\frac{\partial^{2} S}{\partial p^{2}}\right)_{0},
$$

and this leaves us with

$$
\dot{S}=\left[\begin{array}{ll}
\Delta q & \Delta p
\end{array}\right]\left[\begin{array}{ll}
A & B \\
B & C
\end{array}\right]\left[\begin{array}{c}
\Delta q \\
\Delta p
\end{array}\right]+\mathcal{O}\left(\Delta^{3}\right) .
$$

Now, imposing the Hamiltonian assumptions H1(a) and H2 [Eqs. (8) and (10), respectively] simplifies $A$ and $C$, from above [Eqs. (19) and (20), respectively] to

$$
\begin{gathered}
A=-\left(\frac{\partial^{2} H}{\partial q^{2}}\right)_{0}\left(\frac{\partial^{2} S}{\partial q \partial p}\right)_{0}, \\
C=\left(\frac{\partial^{2} H}{\partial p^{2}}\right)_{0}\left(\frac{\partial^{2} S}{\partial p \partial q}\right)_{0} .
\end{gathered}
$$

Positive definiteness of $(\operatorname{Hess}(\dot{S}))_{0}$ (assumption S2) implies that the quadratic form above [Eq. (26)] should be positive definite. This means that we cannot have $\left(\partial^{2} H / \partial p^{2}\right)_{0}=0$, since then $C$ would not be strictly positive and we would get a contradiction. This, combined with assumption H1(b) [Eq. (9)], implies that $\left(\partial^{2} H / \partial p^{2}\right)_{0}>0$. This in combination with $C>0$ means that $\left(\partial^{2} S / \partial p \partial q\right)_{0}>0$. But $A>0$ also, in order to have positive definiteness of the quadratic form [Eq. (26)], and this combined with assumption H3 [Eq. (11)]; i.e. $\left(\partial^{2} H / \partial q^{2}\right)_{0} \geq 0$, implies $\left(\partial^{2} S / \partial p \partial q\right)_{0}<0$. Thus, we get a contradiction, and so no such function $S$ exists.

General N: The extension of the proof to general $N$ follows similar lines, though with a few added subtleties. Let us now proceed with it. As before, suppose $S: \mathscr{P} \rightarrow \mathbb{R}$ is any function on $\mathscr{P}$ satisfying $\mathrm{S} 1$ and $\mathrm{S} 2$. Its time derivative at any point $x=\left(q_{1}, \ldots, q_{N}, p_{1}, \ldots, p_{N}\right) \in \mathscr{P}$ can be evaluated via the Poisson bracket:

$$
\dot{S}=\sum_{k=1}^{N}\left(\frac{\partial H}{\partial p_{k}} \frac{\partial S}{\partial q_{k}}-\frac{\partial H}{\partial q_{k}} \frac{\partial S}{\partial p_{k}}\right) .
$$

Let us now insert into this the Taylor series for each term expanded about the equilibrium point $x_{0}=\left(\left(q_{0}\right)_{1}, \ldots\right.$, $\left.\left(q_{0}\right)_{N},\left(p_{0}\right)_{1}, \ldots,\left(p_{0}\right)_{1}\right)$. Denoting $\Delta q_{i}=q_{i}-\left(q_{0}\right)_{i}$ and $\Delta p_{i}=p_{i}-\left(p_{0}\right)_{i}$, and using $\mathcal{O}\left(\Delta^{n}\right)$ to represent $n$ th-order terms in products of $\Delta q_{i}$ and $\Delta p_{i}$, we have

$$
\begin{aligned}
\frac{\partial H}{\partial q_{k}}= & \left(\frac{\partial H}{\partial q_{k}}\right)_{0} \\
& +\sum_{i=1}^{N}\left[\left(\frac{\partial^{2} H}{\partial q_{i} \partial q_{k}}\right)_{0} \Delta q_{i}+\left(\frac{\partial^{2} H}{\partial p_{i} \partial q_{k}}\right)_{0} \Delta p_{i}\right]+\mathcal{O}\left(\Delta^{2}\right)
\end{aligned}
$$

and similarly for the $p_{k}$ partial of $H$, while 
$\frac{\partial S}{\partial q_{k}}=\sum_{i=1}^{N}\left[\left(\frac{\partial^{2} S}{\partial q_{i} \partial q_{k}}\right)_{0} \Delta q_{i}+\left(\frac{\partial^{2} S}{\partial p_{i} \partial q_{k}}\right)_{0} \Delta p_{i}\right]+\mathcal{O}\left(\Delta^{2}\right)$

and similarly for the $p_{k}$ partial of $S$, where we have used the condition S1 [Eq. (6)] which entails that the zero-order term vanishes. Inserting all Taylor series into the Poisson bracket [Eq. (29)] and collecting terms, we obtain the following result,

$$
\begin{aligned}
\dot{S} & =\left[\begin{array}{ll}
\mathbf{a}^{\mathrm{T}} & \mathbf{b}^{\mathrm{T}}
\end{array}\right]\left[\begin{array}{c}
\Delta q_{1} \\
\vdots \\
\Delta p_{N}
\end{array}\right] \\
& +\left[\begin{array}{lll}
\Delta q_{1} & \cdots & \Delta p_{N}
\end{array}\right]\left[\begin{array}{cc}
\mathbf{A} & \mathbf{B} \\
\mathbf{B}^{\mathrm{T}} & \mathbf{C}
\end{array}\right]\left[\begin{array}{c}
\Delta q_{1} \\
\vdots \\
\Delta p_{N}
\end{array}\right]+\mathcal{O}\left(\Delta^{3}\right),
\end{aligned}
$$

where we have the following components for the $N$-dimensional vectors,

$$
a_{i}=\sum_{k=1}^{N}\left[\left(\frac{\partial H}{\partial p_{k}}\right)_{0}\left(\frac{\partial^{2} S}{\partial q_{i} \partial q_{k}}\right)_{0}-\left(\frac{\partial H}{\partial q_{k}}\right)_{0}\left(\frac{\partial^{2} S}{\partial q_{i} \partial p_{k}}\right)_{0}\right]
$$

and

$$
b_{i}=\sum_{k=1}^{N}\left[\left(\frac{\partial H}{\partial p_{k}}\right)_{0}\left(\frac{\partial^{2} S}{\partial p_{i} \partial q_{k}}\right)_{0}-\left(\frac{\partial H}{\partial q_{k}}\right)_{0}\left(\frac{\partial^{2} S}{\partial p_{i} \partial p_{k}}\right)_{0}\right]
$$

and for the $N \times N$ matrices,

$$
\begin{gathered}
A_{i j}=\frac{1}{2} \sum_{k=1}^{N}\left[\left(\frac{\partial^{2} H}{\partial q_{i} \partial p_{k}}\right)_{0}\left(\frac{\partial^{2} S}{\partial q_{j} \partial q_{k}}\right)_{0}+\left(\frac{\partial^{2} H}{\partial q_{j} \partial p_{k}}\right)_{0}\left(\frac{\partial^{2} S}{\partial q_{i} \partial q_{k}}\right)_{0}-\left(\frac{\partial^{2} H}{\partial q_{i} \partial q_{k}}\right)_{0}\left(\frac{\partial^{2} S}{\partial q_{j} \partial p_{k}}\right)_{0}-\left(\frac{\partial^{2} H}{\partial q_{j} \partial q_{k}}\right)_{0}\left(\frac{\partial^{2} S}{\partial q_{i} \partial p_{k}}\right)_{0}\right], \\
B_{i j}=\frac{1}{2} \sum_{k=1}^{N}\left[\left(\frac{\partial^{2} H}{\partial q_{i} \partial p_{k}}\right)_{0}\left(\frac{\partial^{2} S}{\partial p_{j} \partial q_{k}}\right)_{0}+\left(\frac{\partial^{2} H}{\partial p_{j} \partial p_{k}}\right)_{0}\left(\frac{\partial^{2} S}{\partial q_{i} \partial q_{k}}\right)_{0}-\left(\frac{\partial^{2} H}{\partial q_{i} \partial q_{k}}\right)_{0}\left(\frac{\partial^{2} S}{\partial p_{j} \partial p_{k}}\right)_{0}-\left(\frac{\partial^{2} H}{\partial p_{j} \partial q_{k}}\right)_{0}\left(\frac{\partial^{2} S}{\partial q_{i} \partial p_{k}}\right)_{0}\right],
\end{gathered}
$$

$C_{i j}=\frac{1}{2} \sum_{k=1}^{N}\left[\left(\frac{\partial^{2} H}{\partial p_{i} \partial p_{k}}\right)_{0}\left(\frac{\partial^{2} S}{\partial p_{j} \partial q_{k}}\right)_{0}+\left(\frac{\partial^{2} H}{\partial p_{j} \partial p_{k}}\right)_{0}\left(\frac{\partial^{2} S}{\partial p_{i} \partial q_{k}}\right)_{0}-\left(\frac{\partial^{2} H}{\partial p_{i} \partial q_{k}}\right)_{0}\left(\frac{\partial^{2} S}{\partial p_{j} \partial p_{k}}\right)_{0}-\left(\frac{\partial^{2} H}{\partial p_{j} \partial q_{k}}\right)_{0}\left(\frac{\partial^{2} S}{\partial p_{i} \partial p_{k}}\right)_{0}\right]$.

By assumption $\mathrm{S} 2$, we have that $\dot{S}$ as given above [Eq. (32)] is strictly positive for any $x \neq x_{0}$ in $\mathscr{P}$. In particular, let $\delta>0$, and let us consider $\dot{S}$ [Eq. (32)] evaluated at the sequence of points $\left\{x_{n}^{ \pm}\right\}_{n=1}^{\infty}$, where $x_{n}^{ \pm}=\left(\left(q_{0}\right)_{1}, \ldots,\left(q_{0}\right)_{l} \pm \delta / n, \ldots,\left(q_{0}\right)_{N},\left(p_{0}\right)_{1}, \ldots,\left(p_{0}\right)_{1}\right)$, for any $l$, such that the only deviation away from equilibrium is along the direction $\Delta q_{l}= \pm \delta / n$, with all other $\Delta q_{i}$ and $\Delta p_{i}$ vanishing. Then, for any $n$, we must have according to the above expression for $\dot{S}$ [Eq. (32)]

$$
\begin{gathered}
\dot{S}\left(x_{n}^{+}\right)=a_{l} \frac{\delta}{n}+A_{l l} \frac{\delta^{2}}{n^{2}}+\mathcal{O}\left(\frac{\delta^{3}}{n^{3}}\right)>0, \\
\dot{S}\left(x_{n}^{-}\right)=-a_{l} \frac{\delta}{n}+A_{l l} \frac{\delta^{2}}{n^{2}}+\mathcal{O}\left(\frac{\delta^{3}}{n^{3}}\right)>0 .
\end{gathered}
$$

Taking the $n \rightarrow \infty$ limit of the first inequality implies $a_{l} \geq 0$, while doing the same for the second inequality implies $a_{l} \leq 0$. Hence, $a_{l}=0$. Since $l$ is arbitrary, this means that $a_{i}=0, \forall i$. A similar argument (using $\left.\Delta p_{l}= \pm \delta / n\right)$ implies $b_{i}=0, \forall i$. Thus, $S$ needs to satisfy the constraints

$0=\sum_{k=1}^{N}\left[\left(\frac{\partial H}{\partial p_{k}}\right)_{0}\left(\frac{\partial^{2} S}{\partial q_{i} \partial q_{k}}\right)_{0}-\left(\frac{\partial H}{\partial q_{k}}\right)_{0}\left(\frac{\partial^{2} S}{\partial q_{i} \partial p_{k}}\right)_{0}\right]$

$$
0=\sum_{k=1}^{N}\left[\left(\frac{\partial H}{\partial p_{k}}\right)_{0}\left(\frac{\partial^{2} S}{\partial p_{i} \partial q_{k}}\right)_{0}-\left(\frac{\partial H}{\partial q_{k}}\right)_{0}\left(\frac{\partial^{2} S}{\partial p_{i} \partial p_{k}}\right)_{0}\right],
$$


and this leaves us with

$$
\dot{S}=\left[\begin{array}{lll}
\Delta q_{1} & \cdots & \Delta p_{N}
\end{array}\right]\left[\begin{array}{cc}
\mathbf{A} & \mathbf{B} \\
\mathbf{B}^{\mathrm{T}} & \mathbf{C}
\end{array}\right]\left[\begin{array}{c}
\Delta q_{1} \\
\vdots \\
\Delta p_{N}
\end{array}\right]+\mathcal{O}\left(\Delta^{3}\right) .
$$

Now, imposing the Hamiltonian assumptions H1(a) and H2 [Eqs. (8) and (10), respectively] simplifies $\mathbf{A}$ and $\mathbf{C}$, from the above [Eqs. (35) and (37), respectively] to

$$
\begin{aligned}
A_{i j}= & -\frac{1}{2} \sum_{k=1}^{N}\left[\left(\frac{\partial^{2} H}{\partial q_{i} \partial q_{k}}\right)_{0}\left(\frac{\partial^{2} S}{\partial q_{j} \partial p_{k}}\right)_{0}\right. \\
& \left.+\left(\frac{\partial^{2} H}{\partial q_{j} \partial q_{k}}\right)_{0}\left(\frac{\partial^{2} S}{\partial q_{i} \partial p_{k}}\right)_{0}\right], \\
C_{i j}= & \frac{1}{2}\left[\left(\frac{\partial^{2} H}{\partial p_{i}^{2}}\right)_{0}\left(\frac{\partial^{2} S}{\partial p_{j} \partial q_{i}}\right)_{0}+\left(\frac{\partial^{2} H}{\partial p_{j}^{2}}\right)_{0}\left(\frac{\partial^{2} S}{\partial p_{i} \partial q_{j}}\right)_{0}\right] .
\end{aligned}
$$

Positive definiteness of $(\operatorname{Hess}(\dot{S}))_{0}$ implies that the quadratic form above [Eq. (42)] should be positive definite. This means that we cannot have $\left(\partial^{2} H / \partial p_{j}^{2}\right)_{0}=0, \forall j$, since then $\mathbf{C}$ would not be positive definite and we would get a contradiction. This, combined with assumption $\mathrm{H} 1(\mathrm{~b})$ [Eq. (9)], implies that $\left(\partial^{2} H / \partial p_{j}^{2}\right)_{0}>0, \forall j$. Moreover, we also have

$$
\sum_{i, j=1}^{N} C_{i j}=\sum_{i, j=1}^{N}\left(\frac{\partial^{2} H}{\partial p_{j}^{2}}\right)_{0}\left(\frac{\partial^{2} S}{\partial p_{i} \partial q_{j}}\right)_{0}>0 .
$$

The reason for this is easily seen by noting that positive definiteness of $\mathbf{C}$, by definition, means that its product with any nonzero vector and its transpose should be positive, i.e. $\mathbf{z}^{\mathrm{T}} \mathbf{C z}>0$ for any nonzero vector $\mathbf{z}$; in particular, $\mathbf{z}=$ $(1,1, \ldots, 1)^{\mathrm{T}}$ achieves the above inequality [Eq. (46)]. But then, let us consider $\sum_{i, j=1}^{N} A_{i j}$. Positive definiteness of $(\operatorname{Hess}(\dot{S}))_{0}$ (i.e. of the quadratic form [Eq. (32)]) implies, just as in the case of $\mathbf{C}$, that $\sum_{i, j=1}^{N} A_{i j}>0$ or

$$
\sum_{i, j=1}^{N}\left(-A_{i j}\right)<0 .
$$

At the same time, we have

$$
\sum_{i, j=1}^{N}\left(-A_{i j}\right)=\sum_{i, j, k=1}^{N}\left(\frac{\partial^{2} H}{\partial q_{i} \partial q_{k}}\right)_{0}\left(\frac{\partial^{2} S}{\partial q_{j} \partial p_{k}}\right)_{0} .
$$

Taking the minimum over the $k$ index in the term with the $H$ partials,

$$
\sum_{i, j=1}^{N}\left(-A_{i j}\right) \geq \sum_{i, j, k=1}^{N}\left[\min _{1 \leq l \leq N}\left(\frac{\partial^{2} H}{\partial q_{i} \partial q_{l}}\right)_{0}\right]\left(\frac{\partial^{2} S}{\partial q_{j} \partial p_{k}}\right)_{0} .
$$

This means that the sums can be separated, and after relabelling, the above [Eq. (49)] becomes

$\sum_{i, j=1}^{N}\left(-A_{i j}\right) \geq\left[\min _{1 \leq l \leq N} \sum_{k=1}^{N}\left(\frac{\partial^{2} H}{\partial q_{k} \partial q_{l}}\right)_{0}\right] \sum_{i, j=1}^{N}\left(\frac{\partial^{2} S}{\partial p_{i} \partial q_{j}}\right)_{0}$.

Now, insert the identity $1=\left(\partial^{2} H / \partial p_{j}^{2}\right)_{0} /\left(\partial^{2} H / \partial p_{j}^{2}\right)_{0}$ into the $i, j$ sum, and maximize over the denominator to get

$$
\begin{aligned}
\sum_{i, j=1}^{N}\left(-A_{i j}\right) & \geq\left[\min _{1 \leq l \leq N} \sum_{k=1}^{N}\left(\frac{\partial^{2} H}{\partial q_{k} \partial q_{l}}\right)_{0}\right] \sum_{i, j=1}^{N} \frac{\left(\partial^{2} H / \partial p_{j}^{2}\right)_{0}}{\left(\partial^{2} H / \partial p_{j}^{2}\right)_{0}}\left(\frac{\partial^{2} S}{\partial p_{i} \partial q_{j}}\right)_{0} \\
& \geq\left[\min _{1 \leq l \leq N} \sum_{k=1}^{N}\left(\frac{\partial^{2} H}{\partial q_{k} \partial q_{l}}\right)_{0}\right] \sum_{i, j=1}^{N}\left[\max _{1 \leq m \leq N}\left(\frac{\partial^{2} H}{\partial p_{m}^{2}}\right)_{0}\right]^{-1}\left(\frac{\partial^{2} H}{\partial p_{j}^{2}}\right)_{0}\left(\frac{\partial^{2} S}{\partial p_{i} \partial q_{j}}\right)_{0} \\
& =\left\{\left[\min _{1 \leq l \leq N} \sum_{k=1}^{N}\left(\frac{\partial^{2} H}{\partial q_{k} \partial q_{l}}\right)_{0}\right]\left[\max _{1 \leq m \leq N}\left(\frac{\partial^{2} H}{\partial p_{m}^{2}}\right)_{0}\right]^{-1}\right\} \sum_{i, j=1}^{N} C_{i j} \\
& \geq 0
\end{aligned}
$$

since the term in curly brackets is non-negative (because of assumption $\mathrm{H} 3$ on the Hamiltonian), and we had earlier $\sum_{i, j=1}^{N} C_{i j}>0$. But we also had $\sum_{i, j=1}^{N}\left(-A_{i j}\right)<0$. Hence, we get a contradiction. Therefore, no such function $S$ exists. This concludes our proof. 


\section{Topological approach}

We now turn to the topological approach to answering Problem I in CM. First, we review the basic ideas of Olsen's line of argumentation [23], and then we discuss their connections with the periodicity of phase space orbits.

\section{Review of Olsen's proof}

The assumptions made on $S: \mathscr{P} \rightarrow \mathbb{R}$ are in this case not as strict as in the perturbative approach. See Fig. 2 for a pictorial representation.

In effect, we simply need to assume that $S$ is nondecreasing along trajectories, which are confined to an invariant closed space $\mathscr{P}$. Under these conditions, Olsen furnishes two proofs [23] for why $S$ is necessarily a constant. In the first one, the essential idea is that the volume integral of $S$ in $\mathscr{P}$ can be written after a change of variables as

$$
\int_{\mathscr{P}} \Omega S=\int_{\mathscr{P}} \Omega\left(S \circ \Phi_{t}\right)
$$

owing to the fact that $\mathscr{P}$ is left invariant by the Hamiltonian flow $\Phi_{t}$ generated by the Hamiltonian vector field $\boldsymbol{X}_{H}$ and that $\mathcal{L}_{X_{H}} \Omega=0$. Because the above expression [Eq. (55)] is time independent, $S$ must be time independent, and hence constant along trajectories. The second proof (based on the same assumptions) is rather more technical but relies also basically on topological ideas; in fact, it is more related to the Poincaré recurrence property [19].

We can make a few remarks. First, there is in this case no requirement on the specific form of the Hamiltonian function $H: \mathscr{P} \rightarrow \mathbb{R}$. In fact, $H$ can even contain explicit dependence on time, and the proof still holds.

Second, the essential ingredient here is the compactness of the phase space $\mathscr{P}$. Indeed, even in Poincaré's original recurrence theorem [13] (as per our discussion in Appendix A), the only necessary assumptions were also phase space compactness and invariance along with Liouville's theorem.

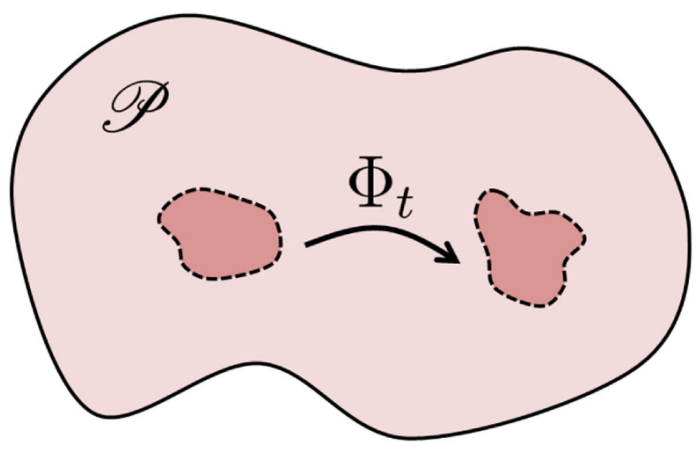

FIG. 2. The topological approach relies on phase space compactness and Liouville's theorem, i.e. the fact that the Hamiltonian flow is volume preserving.

\section{Periodicity in phase space}

Even more can be said about the connection between phase space compactness and the recurrence of orbits than the Poincaré recurrence theorem. There are recent theorems in symplectic geometry which show that exact periodicity of orbits can exist in compact phase spaces.

For example, let us assume the Hamiltonian is of typical form [Eq. (12)]. Then, there is a theorem [38] which states that for a compact configuration space $\mathscr{Q}$ we have periodic solutions of $\boldsymbol{X}_{H}$. In fact, it was even shown [39] that we have periodic solutions provided certain conditions on the potential $V$ are satisfied and $\mathscr{Q}$ just needs to have bounded geometry (i.e. to be geodesically complete and to have the scalar curvature and derivative thereof bounded).

Thus, under the assumption of compactness or any other condition which entails closed orbits, we cannot have a function which behaves like entropy in this sense for a very simple reason. Assume $S: \mathscr{P} \rightarrow \mathbb{R}$ is nondecreasing along trajectories, and let us consider an orbit $\gamma: \mathbb{R} \rightarrow \mathscr{P}$ in phase space [satisfying $\mathrm{d} \gamma(t) / \mathrm{d} t=$ $\left.\boldsymbol{X}_{H}(\gamma(t))\right]$ which is closed. This means that for any $x \in \mathscr{P}$ on the orbit there exist $t_{0}, T \in \mathbb{R}$ such that $x=\gamma\left(t_{0}\right)=\gamma\left(t_{0}+T\right)$. Hence, we have $S(x)=S\left(\gamma\left(t_{0}\right)\right)=$ $S\left(\gamma\left(t_{0}+T\right)\right)=S(x)$, so $S$ is constant along the orbit and therefore cannot behave like entropy.

\section{ENTROPY THEOREMS IN GENERAL RELATIVITY}

We now turn to address the question of why these theorems do not carry over from CM to GR.

\section{A. Setup}

Let $\mathscr{M}$ be a 4-manifold with a Lorentzian metric $g$ of signature $(-,+,+,+)$ and having a metric-compatible derivative operator $\nabla$. We choose a coordinate system where $t$ denotes the time coordinate, and $\boldsymbol{t}$ is the time flow vector field on $\mathscr{M}$. We label $\Sigma$ the $t=$ const spacelike 3 -surfaces foliating $\mathscr{M}$, such that $\mathscr{M}=\mathbb{R} \times \Sigma$. On $\Sigma$, we have an induced three-dimensional Riemannian metric $\boldsymbol{h}$, with a metric-compatible derivative operator $D$. Its conjugate momentum is $\boldsymbol{\pi}=\sqrt{h}(\boldsymbol{K}-\boldsymbol{K} \boldsymbol{h})$, where $h=\operatorname{det}(\boldsymbol{h}), \boldsymbol{K}$ is the extrinsic curvature of $\Sigma$, and $K=\operatorname{tr}(\boldsymbol{K})$. Moreover, let $N$ and $N$, respectively, be the lapse function and shift vector of $g$.

Consider any field theory on $\mathscr{M}$, in this case described by an infinite-dimensional phase space $\mathscr{P}$. Let us write, in general, any point in phase space as $(\varphi, \pi) \in \mathscr{P}$, where $\pi=\left\{\pi_{A}(x)\right\}$ is the set of momenta canonically conjugate to the fields $\varphi=\left\{\varphi_{A}(x)\right\}$ and $A$ is a general (possibly multi-)index for the fields. For any functional $F: \mathscr{P} \rightarrow \mathbb{R}$, we can compute its time derivative $\dot{F}=$ $\mathcal{L}_{t} F=\{F, H\}$ via a suitably defined Poisson bracket with the Hamiltonian $H: \mathscr{P} \rightarrow \mathbb{R}$, which takes an 
analogous form as that in $\mathrm{CM}$ but expressed in terms of functional derivatives [35]:

$\dot{F}=\int_{\Sigma} \mathrm{d}^{3} x \sum_{A}\left(\frac{\delta H}{\delta \pi_{A}(x)} \frac{\delta F}{\delta \varphi_{A}(x)}-\frac{\delta H}{\delta \varphi_{A}(x)} \frac{\delta F}{\delta \pi_{A}(x)}\right)$.

In particular, we will have canonical equations of motion $\dot{\varphi}_{A}=\left\{\varphi_{A}, H\right\}$ and $\dot{\pi}_{A}=\left\{\pi_{A}, H\right\}$.

For defining and working with differential forms on $\mathscr{P}$, we also require a notion of an exterior derivative. Because we are dealing with field theories, we must use the functional exterior derivative $\delta$ (see Refs. [40,41] for more details): for example, $\delta \varphi_{A}(x)$ is a 1 -form on $\mathscr{P}$, and so for any functional (zero-form) $\Gamma\left[\varphi_{A}(x)\right]$ on $\mathscr{P}$, for example, we have that the action of $\delta$ is given by $\delta \Gamma\left[\varphi_{A}(x)\right]=\int_{\Sigma} \mathrm{d}^{3} x\left(\delta \Gamma / \delta \varphi_{A}(x)\right) \delta \varphi_{A}(x)$. This can then be extended to also define $p$-forms.

\section{B. Perturbative approach}

We wish to investigate under what conditions the CM no-entropy proof of Sec. III B transfers over to field theories in curved spacetime. To this effect, we consider the equivalent setup: broadly speaking, we ask whether there exists a phase space functional $S: \mathscr{P} \rightarrow \mathbb{R}$ which is increasing in time everywhere except at an equilibrium configuration. In particular, we use the following two entropy conditions in analogy with those of Sec. III B 3 in $\mathrm{CM}$ :

S1 (existence of equilibrium): We assume there exists a point $x_{0}=(\stackrel{\circ}{\varphi}, \stackrel{\circ}{\pi}) \in \mathscr{P}$, where $S$ is stationary and (to simplify the analysis) $H$ is stationary as well:

$$
\frac{\delta S[\stackrel{\circ}{\varphi}, \stackrel{\circ}{\pi}]}{\delta \stackrel{\circ}{\varphi}_{A}(x)}=\frac{\delta S[\stackrel{\circ}{\varphi}, \stackrel{\circ}{\pi}]}{\delta \stackrel{\circ}{\pi}_{A}(x)}=0=\frac{\delta H[\stackrel{\circ}{\varphi}, \stackrel{\circ}{\pi}]}{\delta \stackrel{\circ}{\varphi}_{A}(x)}=\frac{\delta H[\stackrel{\circ}{\varphi}, \stackrel{\circ}{\pi}]}{\delta \stackrel{\circ}{\pi}_{A}(x)} .
$$

This implies $\dot{S}[\stackrel{\circ}{\varphi}, \stackrel{\circ}{\pi}]=0=\dot{H}[\stackrel{\circ}{\varphi}, \stackrel{\circ}{\pi}]$.

S2 (second law of thermodynamics): We assume that the Hessian of $\dot{S}$ is positive definite at equilibrium, i.e. $\operatorname{Hess}(\dot{S}[\stackrel{\circ}{\varphi}, \stackrel{\circ}{\pi}]) \succ 0$. This is a sufficient condition to ensure that $\dot{S}>0$ in $\mathscr{P} \backslash x_{0}$, and $\dot{S}=0$ at $x_{0}$.

We then follow the same procedure as in Sec. III B 4: we insert into the Poisson bracket

$\dot{S}=\int_{\Sigma} \mathrm{d}^{3} x \sum_{A}\left(\frac{\delta H[\varphi, \pi]}{\delta \pi_{A}(x)} \frac{\delta S[\varphi, \pi]}{\delta \varphi_{A}(x)}-\frac{\delta H[\varphi, \pi]}{\delta \varphi_{A}(x)} \frac{\delta S[\varphi, \pi]}{\delta \pi_{A}(x)}\right)$

the functional Taylor series [42] for each term about $(\stackrel{\circ}{\varphi}, \stackrel{\circ}{\pi})$, denoting $\Delta \varphi_{A}(x)=\varphi_{A}(x)-\stackrel{\circ}{\varphi}(x)$ and $\Delta \pi_{A}(x)=$ $\pi_{A}(x)-\stackrel{\circ}{\pi}(x)$,

$$
\begin{aligned}
\frac{\delta H[\varphi, \pi]}{\delta \pi_{A}(x)}= & \frac{\delta H[\stackrel{\circ}{\varphi}, \stackrel{\circ}{\pi}]}{\delta \stackrel{\circ}{\pi}_{A}(x)}+\int_{\Sigma} \mathrm{d}^{3} y \sum_{B}\left\{\frac{\delta^{2} H[\stackrel{\circ}{\varphi}, \stackrel{\circ}{\pi}]}{\delta \stackrel{\circ}{\varphi}_{B}(y) \delta \stackrel{\circ}{\pi}_{A}(x)} \Delta \stackrel{\circ}{\varphi}_{B}(y)\right. \\
& \left.+\frac{\delta^{2} H[\stackrel{\circ}{\varphi}, \stackrel{\circ}{\pi}]}{\delta \stackrel{\circ}{\pi}_{B}(y) \delta \stackrel{\circ}{\pi}_{A}(x)} \Delta \stackrel{\circ}{\pi}_{B}(y)\right\}+\mathcal{O}\left(\Delta^{2}\right)
\end{aligned}
$$

and similarly for the other terms. Then, we apply S1 in this case [Eq. (57)], which makes all zero-order terms vanish. Finally, the Poisson bracket in this case [Eq. (58)] becomes

$$
\begin{aligned}
& \dot{S}=\int_{\Sigma} \mathrm{d}^{3} x \int_{\Sigma} \mathrm{d}^{3} y \int_{\Sigma} \mathrm{d}^{3} z \sum_{A, B, C}\left\{\left[\frac{\delta^{2} H[\stackrel{\circ}{\varphi}, \stackrel{\circ}{\pi}]}{\delta \stackrel{\circ}{\varphi}_{B}(y) \delta \stackrel{\circ}{\pi}_{A}(x)} \frac{\delta^{2} S[\stackrel{\circ}{\varphi}, \stackrel{\circ}{\pi}]}{\delta \stackrel{\circ}{\varphi}_{C}(z) \delta \stackrel{\circ}{\varphi}_{A}(x)}-\frac{\delta^{2} H[\stackrel{\circ}{\varphi}, \stackrel{\circ}{\pi}]}{\delta \stackrel{\circ}{\varphi}_{B}(y) \delta \stackrel{\circ}{\varphi}_{A}(x)} \frac{\delta^{2} S[\stackrel{\circ}{\varphi}, \stackrel{\circ}{\pi}]}{\delta \stackrel{\circ}{\varphi}_{C}(z) \delta \stackrel{\circ}{\pi}_{A}(x)}\right] \Delta \varphi_{B}(y) \Delta \varphi_{C}(z)\right. \\
& +\left[\frac{\delta^{2} H[\stackrel{\circ}{\varphi}, \stackrel{\circ}{\pi}]}{\delta \stackrel{\circ}{\varphi}_{B}(y) \delta \stackrel{\circ}{\pi}_{A}(x)} \frac{\delta^{2} S[\stackrel{\circ}{\varphi}, \stackrel{\circ}{\pi}]}{\delta \stackrel{\circ}{\pi}_{C}(z) \delta \stackrel{\circ}{\varphi}_{A}(x)}+\frac{\delta^{2} H[\stackrel{\circ}{\varphi}, \stackrel{\circ}{\pi}]}{\delta \stackrel{\circ}{\pi}_{C}(z) \delta \stackrel{\circ}{\pi}_{A}(x)} \frac{\delta^{2} S[\stackrel{\circ}{\varphi}, \stackrel{\circ}{\pi}]}{\delta \stackrel{\circ}{\varphi}_{B}(y) \delta \stackrel{\circ}{\varphi}_{A}(x)}\right. \\
& \left.-\frac{\delta^{2} H[\stackrel{\circ}{\varphi}, \stackrel{\circ}{\pi}]}{\delta \stackrel{\circ}{\varphi}_{B}(y) \delta \stackrel{\circ}{\varphi}_{A}(x)} \frac{\delta^{2} S[\stackrel{\circ}{\varphi}, \stackrel{\circ}{\pi}]}{\delta \stackrel{\circ}{\pi}_{C}(z) \delta \stackrel{\circ}{\pi}_{A}(x)}-\frac{\delta^{2} H[\stackrel{\circ}{\varphi}, \stackrel{\circ}{\pi}]}{\delta \stackrel{\circ}{\pi}_{C}(z) \delta \stackrel{\circ}{\varphi}_{A}(x)} \frac{\delta^{2} S[\stackrel{\circ}{\varphi}, \stackrel{\circ}{\pi}]}{\delta \stackrel{\circ}{\varphi}_{B}(y) \delta \stackrel{\circ}{\pi}_{A}(x)}\right] \Delta \varphi_{B}(y) \Delta \pi_{C}(z) \\
& \left.+\left[\frac{\delta^{2} H[\stackrel{\circ}{\varphi}, \stackrel{\circ}{\pi}]}{\delta \stackrel{\circ}{\pi}_{B}(y) \delta \stackrel{\circ}{\pi}_{A}(x)} \frac{\delta^{2} S[\stackrel{\circ}{\varphi}, \stackrel{\circ}{\pi}]}{\delta \stackrel{\circ}{\pi}_{C}(z) \delta \stackrel{\circ}{\varphi}_{A}(x)}-\frac{\delta^{2} H[\stackrel{\circ}{\varphi}, \stackrel{\circ}{\pi}]}{\delta \stackrel{\circ}{\pi}_{B}(y) \delta \stackrel{\circ}{\varphi}_{A}(x)} \frac{\delta^{2} S[\stackrel{\circ}{\varphi}, \stackrel{\circ}{\pi}]}{\delta \stackrel{\circ}{\pi}_{C}(z) \delta \stackrel{\circ}{\pi}_{A}(x)}\right] \Delta \pi_{B}(y) \Delta \pi_{C}(z)\right\}+\mathcal{O}\left(\Delta^{3}\right) .
\end{aligned}
$$

We compute this, in turn, for a scalar field in curved spacetime, for EM in curved spacetime, and for GR. We will show that no function $S$ obeying the conditions $\mathrm{S} 1$ and $\mathrm{S} 2$ given here exists in the case of the first two but that the same cannot be said of the latter.

\section{Scalar field}

Let us consider a theory for a scalar field $\phi(x)$ in a potential $V[\phi(x)]$, defined by the Lagrangian

$$
L=\sqrt{-g}\left(-\frac{1}{2} g^{a b} \nabla_{a} \phi \nabla_{b} \phi-V[\phi]\right) .
$$


There are no constraints in this case. For turning the above [Eq. (61)] into a canonical theory, let us choose a foliation of $\mathscr{M}$ such that $\boldsymbol{N}=0$. The canonical measure [41] is then simply given by $\Omega=\int_{\Sigma} \mathrm{d}^{3} x \delta \dot{\phi} \wedge \delta \phi$, and the Hamiltonian [43] is

$H[\phi, \pi]=\int_{\Sigma} \mathrm{d}^{3} x N\left(\frac{\pi^{2}}{2 \sqrt{h}}+\frac{\sqrt{h}}{2} h^{a b} \nabla_{a} \phi \nabla_{b} \phi+\sqrt{h} V[\phi]\right)$,

where $\pi=(\sqrt{h} / N) \dot{\phi}$ is the canonical momentum.

Let us compute the second functional derivatives of $H$. We have

$$
\begin{aligned}
\frac{\delta^{2} H[\phi, \pi]}{\delta \phi(y) \delta \phi(x)}= & N(x) \sqrt{h(x)} V^{\prime \prime}[\phi(x)] \delta(x-y) \\
& -\partial_{a}\left(N(x) \sqrt{h(x)} h^{a b}(x) \partial_{b} \delta(x-y)\right),
\end{aligned}
$$

$$
\frac{\delta^{2} H[\phi, \pi]}{\delta \pi(y) \delta \pi(x)}=\frac{N(x)}{\sqrt{h(x)}} \delta(x-y),
$$

and the mixed derivatives $\delta^{2} H[\phi, \pi] / \delta \pi(y) \delta \phi(x)$ vanish.

We now proceed as outlined above: we assume there exists an entropy function $S: \mathscr{P} \rightarrow \mathbb{R}$ obeying $\mathrm{S} 1$ and $\mathrm{S} 2$ with an equilibrium field configuration $\left(\dot{\phi}, \frac{\AA}{\pi}\right)$, and we will show that there is a contradiction with $\dot{S}>0$.

Additionally, we assume that $V^{\prime \prime}[\stackrel{\phi}{\phi}] \geq 0$; in other words, the equilibrium field configuration is one where the potential is concave upward; i.e. it is a stable equilibrium.

According to the above expression for $\dot{S}$ [Eq. (60)], we have that entropy production in this case is given by

$$
\begin{aligned}
& \dot{S}=\int_{\Sigma} \mathrm{d}^{3} x \mathrm{~d}^{3} y \mathrm{~d}^{3} z\left\{\left[-\frac{\delta^{2} H[\stackrel{\circ}{\phi}, \stackrel{\circ}{\pi}]}{\delta \dot{\circ}(y) \delta \dot{\phi}(x)} \frac{\delta^{2} S[\stackrel{\circ}{\phi}, \stackrel{\circ}{\pi}]}{\delta \dot{\phi}(z) \delta \stackrel{\circ}{\pi}(x)}\right] \Delta \phi(y) \Delta \phi(z)+\left[\frac{\delta^{2} H[\stackrel{\circ}{\phi}, \stackrel{\circ}{\pi}]}{\delta \dot{\circ}(z) \delta \stackrel{\circ}{\pi}(x)} \frac{\delta^{2} S[\stackrel{\circ}{\phi}, \stackrel{\circ}{\pi}]}{\delta \dot{\phi}(y) \delta \dot{\phi}(x)}-\frac{\delta^{2} H[\stackrel{\circ}{\phi}, \stackrel{\circ}{\pi}]}{\delta \dot{\phi}(y) \delta \dot{\phi}(x)} \frac{\delta^{2} S[\stackrel{\circ}{\phi}, \stackrel{\circ}{\pi}]}{\delta \pi(z) \delta(x)}\right]\right. \\
& \left.\times \Delta \phi(y) \Delta \pi(z)+\left[\frac{\delta^{2} H[\stackrel{\circ}{\phi}, \stackrel{\circ}{\pi}]}{\delta \dot{\pi}(y) \delta \stackrel{\circ}{\pi}(x)} \frac{\delta^{2} S[\stackrel{\circ}{\phi}, \stackrel{\circ}{\pi}]}{\delta \stackrel{\circ}{\pi}(z) \delta \dot{\phi}(x)}\right] \Delta \pi(y) \Delta \pi(z)\right\}+\mathcal{O}\left(\Delta^{3}\right),
\end{aligned}
$$

where we have used the fact that the mixed derivatives vanish. Let us now evaluate $\dot{S}$ along different directions in $\mathscr{P}$ away from $(\dot{\phi}, \stackrel{\pi}{\pi})$. Suppose $\Delta \pi$ is nonzero everywhere on $\Sigma$ and $\Delta \phi$ vanishes everywhere on $\Sigma$. Then, using the second momentum derivative of $H$ [Eq. (64)], $\dot{S}$ [Eq. (65)] becomes

$$
\begin{gathered}
\dot{S}=\int_{\Sigma} \mathrm{d}^{3} x \mathrm{~d}^{3} y \mathrm{~d}^{3} z \frac{N(x)}{\sqrt{h(x)}} \delta(x-y) \\
\times \frac{\delta^{2} S[\stackrel{\circ}{\phi}, \stackrel{\circ}{\pi}]}{\delta \stackrel{\circ}{\pi}(z) \delta \dot{\phi}(x)} \Delta \pi(y) \Delta \pi(z)+\mathcal{O}\left(\Delta^{3}\right) \\
=\int_{\Sigma} \mathrm{d}^{3} y \mathrm{~d}^{3} z \frac{N(y)}{\sqrt{h(y)}} \frac{\delta^{2} S[\stackrel{\circ}{\phi}, \stackrel{\circ}{\pi}]}{\delta \stackrel{\circ}{\pi}(z) \delta \stackrel{\circ}{\phi}(y)} \Delta \pi(y) \Delta \pi(z)+\mathcal{O}\left(\Delta^{3}\right) \\
\leq\left\{\max _{x \in \Sigma} \frac{N(x)}{\sqrt{h(x)}}(\Delta \pi(x))^{2}\right\} \int_{\Sigma} \mathrm{d}^{3} y \mathrm{~d}^{3} z \frac{\delta^{2} S[\stackrel{\circ}{\phi}, \stackrel{\circ}{\pi}]}{\delta \stackrel{\circ}{\pi}(z) \delta \dot{\phi}(y)} \\
+\mathcal{O}\left(\Delta^{3}\right) .
\end{gathered}
$$

The requirement that the lhs of the first line above [Eq. (66)] is strictly positive, combined with the strict positivity of the term in curly brackets in the third line [Eq. (68)] and the assumption (S2) of the definiteness of the
Hessian of $\dot{S}$ at $(\dot{\phi}, \stackrel{\pi}{\pi})$, altogether mean that the above [Eqs. (66)-(68)] imply

$$
\int_{\Sigma} \mathrm{d}^{3} y \mathrm{~d}^{3} z \frac{\delta^{2} S[\stackrel{\circ}{\phi}, \stackrel{\circ}{\pi}]}{\delta \stackrel{\circ}{\pi}(z) \delta \dot{\phi}(y)}>0 .
$$

Now, let us evaluate $\dot{S}$ in a region of $\mathscr{P}$ where $\Delta \phi$ is nonzero everywhere on $\Sigma$, while $\Delta \pi$ vanishes everywhere on $\Sigma$. Then, using the second field derivative of $H$ [Eq. (63)], the negative of the above expression for $\dot{S}$ [Eq. (65)] becomes

$$
\begin{aligned}
-\dot{S}= & \int_{\Sigma} \mathrm{d}^{3} x \mathrm{~d}^{3} y \mathrm{~d}^{3} z\left\{N(x) \sqrt{h(x)} V^{\prime \prime}[\stackrel{\circ}{\phi}(x)] \delta(x-y)\right. \\
& \left.-\partial_{a}\left(N(x) \sqrt{h(x)} h^{a b}(x) \partial_{b} \delta(x-y)\right)\right\} \\
& \times \frac{\delta^{2} S[\stackrel{\circ}{\phi}, \stackrel{\circ}{\pi}]}{\delta \dot{\phi}(z) \delta \stackrel{\circ}{\pi}(x)} \Delta \phi(y) \Delta \phi(z)+\mathcal{O}\left(\Delta^{3}\right) .
\end{aligned}
$$

Now, observe that

$$
\begin{gathered}
\int_{\Sigma} \mathrm{d}^{3} x \mathrm{~d}^{3} y \mathrm{~d}^{3} z\left\{\partial_{a}\left(N(x) \sqrt{h(x)} h^{a b}(x) \partial_{b} \delta(x-y)\right)\right\} \\
\times \frac{\delta^{2} S[\stackrel{\circ}{\phi}, \stackrel{\circ}{\pi}]}{\delta \grave{\phi}(z) \delta \stackrel{\circ}{\pi}(x)} \Delta \phi(y) \Delta \phi(z)
\end{gathered}
$$


is simply a boundary term. This can be seen by integrating by parts until the derivative is removed from the delta distribution, the definition of the latter is applied to remove the $x$ integration, and the result is a total derivative in the integrand. Assuming asymptotic decay properties sufficient to make this boundary term vanish, the above $-\dot{S}[$ Eq. (70)] simply becomes

$$
\begin{aligned}
-\dot{S}= & \int_{\Sigma} \mathrm{d}^{3} y \mathrm{~d}^{3} z N(y) \sqrt{h(y)} V^{\prime \prime}[\stackrel{\circ}{\phi}(y)] \\
& \times \frac{\delta^{2} S[\stackrel{\circ}{\phi}, \stackrel{\circ}{\pi}]}{\delta \dot{\phi}(z) \delta \stackrel{\circ}{\pi}(y)} \Delta \phi(y) \Delta \phi(z)+\mathcal{O}\left(\Delta^{3}\right) \\
\geq & \left\{\min _{x \in \Sigma} N(x) \sqrt{h(x)} V^{\prime \prime}[\stackrel{\circ}{\phi}(x)](\Delta \phi(x))^{2}\right\} \\
& \times \int_{\Sigma} \mathrm{d}^{3} y \mathrm{~d}^{3} z \frac{\delta^{2} S[\stackrel{\circ}{\phi}, \stackrel{\circ}{\pi}]}{\delta \dot{\phi}(z) \delta \stackrel{\circ}{\pi}(y)}+\mathcal{O}\left(\Delta^{3}\right) .
\end{aligned}
$$

The lhs of the first line [Eq. (72)] should be strictly negative, and the term in curly brackets in the second line [Eq. (73)] is strictly positive. Hence, owing to the definiteness of the Hessian of $\dot{S}$ at $(\stackrel{\circ}{\phi}, \stackrel{\circ}{\pi})$, and using the symmetry of the arguments in the integrand and equality of mixed derivatives, the above [Eqs. (72) and (73)] imply

$$
\int_{\Sigma} \mathrm{d}^{3} y \mathrm{~d}^{3} z \frac{\delta^{2} S[\stackrel{\circ}{\phi}, \stackrel{\circ}{\pi}]}{\delta \stackrel{\circ}{\pi}(z) \delta \dot{\phi}(y)}<0 .
$$

This is a contradiction with the inequality obtained previously [Eq. (69)]. Therefore, we have no function $S$ for a scalar field theory that behaves like entropy according to assumptions S1 and S2.

We remark that in this case we get the conclusion $\dot{S}=0$ using the perturbative approach despite the fact that the topological one would not work in the case of a noncompact Cauchy surface. The reason is that

$$
\mu(\mathscr{P})=\int_{\mathscr{P}} \Omega=\int_{\mathscr{P}} \int_{\Sigma} \mathrm{d}^{3} x \delta \dot{\phi}(x) \wedge \delta \phi(x)
$$

$$
\begin{aligned}
& \geq \int_{\mathscr{P}} \int_{\Sigma} \mathrm{d}^{3} x \min _{y \in \Sigma}[\delta \dot{\phi}(y) \wedge \delta \phi(y)] \\
= & \int_{\mathscr{P}}\left\{\min _{y \in \Sigma}[\delta \dot{\phi}(y) \wedge \delta \phi(y)]\right\}\left[\int_{\Sigma} \mathrm{d}^{3} x\right],
\end{aligned}
$$

which diverges if $\Sigma$ is noncompact. (Note: The reason why the term in curly brackets is finite but nonzero is that the field and its time derivative cannot be always vanishing at any given point, for if they were it would lead only to the trivial solution.) Thus, only the perturbative approach is useful here for deducing lack of entropy production for spacetimes with noncompact Cauchy surfaces.

\section{Electromagnetism}

Before we inspect EM in curved spacetime, let us carry out the analysis in flat spacetime $(N=1, N=\mathbf{0}$, and $\boldsymbol{h}={ }^{(3)} \boldsymbol{\delta}=\operatorname{diag}(0,1,1,1)$ ), for massive (or de BroglieProca) EM [44], defined by the Lagrangian

$$
L=-\frac{1}{4} \boldsymbol{F}: \boldsymbol{F}-\frac{1}{2} m^{2} \boldsymbol{A} \cdot \boldsymbol{A}+\boldsymbol{A} \cdot \boldsymbol{J}
$$

where $F_{a b}=\partial_{a} A_{b}-\partial_{b} A_{a}, A_{a}$ is the electromagnetic potential and $J_{a}$ is an external source.

We have a constrained Hamiltonian system in this case. In particular, the momentum canonically conjugate to $A_{0}=V$ vanishes identically. This means that, instead of $A_{a}$, we may take (its spatial part) $\mathcal{A}_{a}={ }^{(3)} \delta_{a b} A^{b}$ along with its conjugate momentum, $\pi^{a}=\dot{\mathcal{A}}^{a}-\partial^{a} V$, to be the phase space variables-while appending to the canonical equations of motion resulting from $H[\mathcal{A}, \boldsymbol{\pi}]$ the constraint $0=\delta H / \delta V$. In particular, we have [44]

$$
\begin{aligned}
H[\mathcal{A}, \boldsymbol{\pi}]= & \int_{\Sigma} \mathrm{d}^{3} x\left(\frac{1}{4} \mathcal{F}: \mathcal{F}+\frac{m^{2}}{2}\left(\mathcal{A} \cdot \mathcal{A}-V^{2}\right)-\mathcal{A} \cdot \mathcal{J}\right. \\
& \left.+\frac{1}{2} \pi \cdot \pi-\left(\partial_{a} \pi^{a}+\rho\right) V+\partial_{a}\left(V \pi^{a}\right)\right),
\end{aligned}
$$

where $\mathcal{F}_{a b}={ }^{(3)} \delta_{a c}{ }^{(3)} \delta_{b d} F^{c d}, \rho=J^{0}$, and $\mathcal{J}^{a}={ }^{(3)} \delta^{a b} J_{b}$.

The Poisson bracket [Eq. (60)] is, in this case,

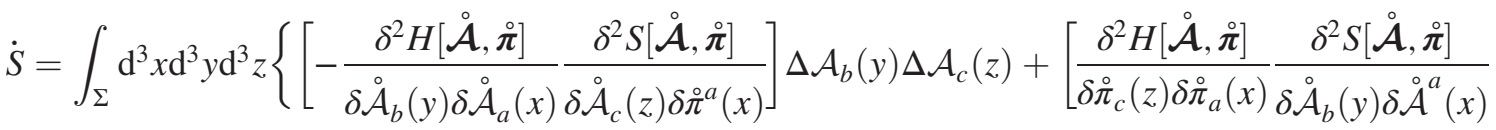

$$
\begin{aligned}
& \left.\left.-\frac{\delta^{2} H[\stackrel{\circ}{\mathcal{A}}, \stackrel{\circ}{\pi}]}{\delta \stackrel{\circ}{\mathcal{A}}_{b}(y) \delta \stackrel{\circ}{\mathcal{A}}_{a}(x)} \frac{\delta^{2} S[\stackrel{\circ}{\mathcal{A}}, \stackrel{\circ}{\pi}]}{\delta \pi_{c}(z) \delta \stackrel{\circ}{\pi}^{a}(x)}\right] \Delta \mathcal{A}_{b}(y) \Delta \pi_{c}(z)+\left[\frac{\delta^{2} H[\stackrel{\circ}{\mathcal{A}}, \stackrel{\circ}{\pi}]}{\delta \stackrel{\circ}{\pi}_{b}(y) \delta \pi_{a}(x)} \frac{\delta^{2} S[\stackrel{\circ}{\mathcal{A},}, \stackrel{\circ}{\pi}]}{\delta \stackrel{\circ}{c}_{c}(z) \delta \stackrel{\circ}{\mathcal{A}}^{a}(x)}\right] \Delta \pi_{b}(y) \Delta \pi_{c}(z)\right\}+\mathcal{O}\left(\Delta^{3}\right),
\end{aligned}
$$

where we have used the fact that the mixed derivatives of the Hamiltonian [Eq. (79)] vanish by inspection and we compute the second field and momentum derivatives thereof to be, respectively, 


$$
\begin{aligned}
& \frac{\delta^{2} H[\stackrel{\circ}{\mathcal{A}}, \stackrel{\circ}{\pi}]}{\delta \stackrel{\circ}{\mathcal{A}}_{b}(y) \delta \stackrel{\circ}{\mathcal{A}}_{a}(x)}=-\left\{{ }^{(3)} \delta^{a b} \partial^{c} \partial_{c} \delta(x-y)-\partial^{b} \partial^{a} \delta(x-y)\right\} \\
& +m^{2}\left[{ }^{(3)} \delta^{a b} \delta(x-y)\right], \\
& \frac{\delta^{2} H[\stackrel{\circ}{\mathcal{A}}, \stackrel{\circ}{\pi}]}{\delta \stackrel{\circ}{\pi}_{b}(y) \delta \AA_{a}^{\circ}(x)}={ }^{(3)} \delta^{a b} \delta(x-y) .
\end{aligned}
$$

Analogously with our strategy in the scalar field case, let us evaluate $\dot{S}$ along different directions away from equilibrium. In particular, let us suppose $\Delta \pi_{1}$ is nonzero everywhere on $\Sigma$ and that $\Delta \pi_{2}, \Delta \pi_{3}$, and $\Delta \mathcal{A}_{a}$ all vanish everywhere on $\Sigma$. Then, using the second momentum derivative of $H$ [Eq. (82)], $\dot{S}$ [Eq. (80)] becomes

$$
\begin{aligned}
& \dot{S}=\int_{\Sigma} \mathrm{d}^{3} x \mathrm{~d}^{3} y \mathrm{~d}^{3} z \delta(x-y) \frac{\delta^{2} S[\stackrel{\circ}{\mathcal{A},}, \stackrel{\circ}{\pi}]}{\delta \stackrel{\circ}{\pi}_{1}(z) \delta \stackrel{\circ}{\mathcal{A}}_{1}(x)} \Delta \pi_{1}(y) \Delta \pi_{1}(z) \\
& +\mathcal{O}\left(\Delta^{3}\right) \\
& =\int_{\Sigma} \mathrm{d}^{3} y \mathrm{~d}^{3} z \frac{\delta^{2} S[\stackrel{\circ}{\mathcal{A}}, \stackrel{\circ}{\boldsymbol{\pi}}]}{\delta \stackrel{\circ}{\pi}_{1}(z) \delta \stackrel{\circ}{\mathcal{A}}_{1}(y)} \Delta \pi_{1}(y) \Delta \pi_{1}(z)+\mathcal{O}\left(\Delta^{3}\right) \\
& \leq\left\{\max _{x \in \Sigma}\left(\Delta \pi_{1}(x)\right)^{2}\right\} \int_{\Sigma} \mathrm{d}^{3} y \mathrm{~d}^{3} z \frac{\delta^{2} S[\stackrel{\circ}{\mathcal{A}}, \stackrel{\circ}{\pi}]}{\delta \stackrel{\circ}{1}_{1}(z) \delta \stackrel{\circ}{\mathcal{A}}_{1}(y)}+\mathcal{O}\left(\Delta^{3}\right) .
\end{aligned}
$$

The argument proceeds as before: the strict positivity of the lhs of the first line above [Eq. (83)] combined with that of the term in curly brackets in the third line [Eq. (85)] and the assumption (S2) of the definiteness of the Hessian of $\dot{S}$ at $(\stackrel{\circ}{\mathcal{A}}, \stackrel{\circ}{\boldsymbol{\pi}})$ altogether mean that the above [Eqs. (83)-(85)] imply

$$
\int_{\Sigma} \mathrm{d}^{3} y \mathrm{~d}^{3} z \frac{\delta^{2} S[\stackrel{\circ}{\mathcal{A}}, \stackrel{\circ}{\pi}]}{\delta \stackrel{\circ}{\pi}_{1}(z) \delta \stackrel{\circ}{\mathcal{A}}_{1}(y)}>0
$$

Now, let us evaluate $\dot{S}$ where $\Delta \mathcal{A}_{1}$ is nonzero everywhere on $\Sigma$, while $\Delta \mathcal{A}_{2}, \Delta \mathcal{A}_{3}$, and $\Delta \pi_{a}$ all vanish everywhere on $\Sigma$. Then, using the second field derivative of $H$ [Eq. (81)], the negative of the above expression for $\dot{S}$ [Eq. (80)] becomes

$$
\begin{aligned}
\dot{S}= & \int_{\Sigma} \mathrm{d}^{3} x \mathrm{~d}^{3} y \mathrm{~d}^{3} z\left[\left(-\left\{{ }^{(3)} \delta^{a b} \partial^{c} \partial_{c} \delta(x-y)\right.\right.\right. \\
& \left.\left.\left.-\partial^{b} \partial^{a} \delta(x-y)\right\}+m^{2}\left[{ }^{(3)} \delta^{a b} \delta(x-y)\right]\right) \frac{\delta^{2} S[\stackrel{\circ}{\mathcal{A}, ~} \boldsymbol{\pi}]}{\delta \dot{\mathcal{A}}_{c}(z) \delta \dot{\AA}^{a}(x)}\right] \\
& \times \Delta \mathcal{A}_{b}(y) \Delta \mathcal{A}_{c}(z)+\mathcal{O}\left(\Delta^{3}\right) .
\end{aligned}
$$

The term in curly brackets simply furnishes a (vanishing) boundary term [up to $\mathcal{O}\left(\Delta^{3}\right)$ ]. Note that for $m=0$ (corresponding to Maxwellian EM in flat spacetime) we would thus get an indefinite Hessian of $\dot{S}$ at $(\stackrel{\circ}{\mathcal{A}}, \stackrel{\circ}{\pi})$, and hence no function $S$ that behaves like entropy as per S1 and S2. So let us assume $m^{2}>0$. Using the symmetry of the arguments in the integrand and equality of mixed derivatives, we are thus left with

$$
\begin{aligned}
-\dot{S} & =\int_{\Sigma} \mathrm{d}^{3} y \mathrm{~d}^{3} z m^{2} \frac{\delta^{2} S[\stackrel{\circ}{\mathcal{A}, \boldsymbol{\pi}]}]}{\delta \dot{\circ}_{1}(z) \delta \stackrel{\circ}{\mathcal{A}}_{1}(y)} \Delta \mathcal{A}_{1}(y) \Delta \mathcal{A}_{1}(z)+\mathcal{O}\left(\Delta^{3}\right) \\
\geq & \left\{m^{2} \min _{x \in \Sigma}\left(\Delta \mathcal{A}_{1}(x)\right)^{2}\right\} \int_{\Sigma} \mathrm{d}^{3} y \mathrm{~d}^{3} z \frac{\delta^{2} S[\stackrel{\circ}{\mathcal{A}}, \stackrel{\circ}{\boldsymbol{\pi}}]}{\delta \pi_{1}(z) \delta \stackrel{\mathcal{A}}{1}_{1}(y)} \\
& +\mathcal{O}\left(\Delta^{3}\right) .
\end{aligned}
$$

The lhs of the first line [Eq. (88)] should be strictly negative, and the term in curly brackets in the second line [Eq. (89)] is strictly positive. Hence, owing to the definiteness of the Hessian of $\dot{S}$ at $(\stackrel{\circ}{\mathcal{A}}, \stackrel{\circ}{\pi})$, the above [Eqs. (88) and (89)] imply

$$
\int_{\Sigma} \mathrm{d}^{3} y \mathrm{~d}^{3} z \frac{\delta^{2} S[\stackrel{\circ}{\mathcal{A}}, \stackrel{\circ}{\pi}]}{\delta \stackrel{\circ}{\pi}_{1}(z) \delta \stackrel{\circ}{\mathcal{A}}_{1}(y)}<0 .
$$

This is a contradiction with the previous inequality on the same quantity [Eq. (86)]. Hence, there is no function $S$ that behaves like entropy (according to S1 and S2) for a massive EM field in flat spacetime.

Let us now carry out the proof for a simple Maxwellian EM field in curved spacetime, defined by the Lagrangian

$$
L=-\frac{1}{4} \sqrt{-g} \boldsymbol{F}: \boldsymbol{F},
$$

where $F_{a b}=\nabla_{a} A_{b}-\nabla_{b} A_{a}$ and $A_{a}$ is the electromagnetic potential. As in the scalar field case, we work with a spacetime foliation such that $N=0$.

As with EM in flat spacetime, this is a constrained Hamiltonian system; the momentum canonically conjugate to $A_{0}=V$ vanishes identically, meaning again that, instead of $A_{a}$, we may take (its spatial part) $\mathcal{A}_{a}=h_{a b} A^{b}$ along with its conjugate momentum, $\pi^{a}=(\sqrt{h} / N) h^{a b}\left(\dot{\mathcal{A}}_{b}-\partial_{b} V\right)$, to be the physical phase space variables-appending to the canonical equations of motion resulting from $H[\mathcal{A}, \boldsymbol{\pi}]$ the constraint $0=\delta H / \delta V=\partial_{a} \pi^{a}$ (which is simply Gauss's law). In particular, we have [44]

$$
H[\mathcal{A}, \boldsymbol{\pi}]=\int_{\Sigma} \mathrm{d}^{3} x\left(\frac{1}{4} N \sqrt{h} \mathcal{F}: \mathcal{F}+\frac{N}{2 \sqrt{h}} \boldsymbol{\pi} \cdot \boldsymbol{\pi}+\pi^{a} \partial_{a} V\right),
$$

where $\mathcal{F}_{a b}=h_{a c} h_{b d} F^{c d}=D_{a} \mathcal{A}_{b}-D_{b} \mathcal{A}_{a}$. 
The Poisson bracket [Eq. (60)] is here given by the same expression as in flat spacetime [Eq. (65)], owing to the fact that the mixed derivatives of the Hamiltonian [Eq. (92)] vanish. Let us focus on regions in phase space where $\Delta \pi$ vanishes everywhere on $\Sigma$ but $\Delta \mathcal{A}$ is everywhere nonzero. There,

$$
\begin{aligned}
\dot{S}= & \int_{\Sigma} \mathrm{d}^{3} x \mathrm{~d}^{3} y \mathrm{~d}^{3} z\left[-\frac{\delta^{2} H[\stackrel{\circ}{\mathcal{A}}, \stackrel{\circ}{\pi}]}{\delta \dot{\mathcal{A}}_{b}(y) \delta \dot{\mathcal{A}}_{a}(x)} \frac{\delta^{2} S[\stackrel{\circ}{\mathcal{A},}, \stackrel{\circ}{\pi}]}{\delta \dot{\mathcal{A}}_{c}(z) \delta \AA^{a}(x)}\right] \\
& \times \Delta \mathcal{A}_{b}(y) \Delta \mathcal{A}_{c}(z)+\mathcal{O}\left(\Delta^{3}\right) .
\end{aligned}
$$

We compute

$$
\begin{aligned}
\frac{\delta^{2} H[\stackrel{\circ}{\mathcal{A}, ~} \boldsymbol{\pi}]}{\delta \stackrel{\circ}{\mathcal{A}}_{b}(y) \delta \stackrel{\circ}{\mathcal{A}}_{a}(x)}= & -\sqrt{h(x)}\left\{D^{c}\left[N(x) h^{a b}(x) D_{c} \delta(x-y)\right]\right. \\
& \left.-D^{b}\left[N(x) D^{a} \delta(x-y)\right]\right\} .
\end{aligned}
$$

Inserting this into the above expression for $\dot{S}$ [Eq. (93)], we simply get a (vanishing) boundary term [up to $\mathcal{O}\left(\Delta^{3}\right)$ ]. We conclude that we have an indefinite Hessian of $\dot{S}$ at $(\stackrel{\circ}{\mathcal{A}}, \stackrel{\circ}{\pi})$, and hence no function $S$ that behaves like entropy as per S1 and $\mathrm{S} 2$.

\section{Gravity}

The phase space $\mathscr{P}$ of GR is, prior to constraint imposition, the space of all Riemannian 3-metrics $\boldsymbol{h}$ on $\Sigma$, together with their corresponding conjugate momenta $\pi$. The symplectic form on $\mathscr{P}$, which is also the volume form, is given by [34]

$$
\Omega=\int_{\Sigma} \mathrm{d}^{3} x \delta \pi^{a b} \wedge \delta h_{a b}
$$

However, concordant with its diffeomorphism invariance, GR is a constrained Hamiltonian system. In particular, the Gauss-Codazzi relations impose certain restrictions on $\boldsymbol{h}$ and $\boldsymbol{\pi}$ that determine which subspaces of $\mathscr{P}$ are dynamically accessible through the canonical equations of motion (as well as being admissible for initial conditions). Indeed, diffeomorphism invariance directly implies that the numerical value of the Hamiltonian should be zero, and therefore that the Hamiltonian functional should simply be a combination of these constraints modulo boundary terms.

Let $\boldsymbol{r}$ be the outward pointing unit normal on $\partial \Sigma$. We denote by $\boldsymbol{\sigma}$ the induced 2-metric on $\partial \Sigma$, with $\sigma=\operatorname{det}(\boldsymbol{\sigma})$, and by $\boldsymbol{k}$ its extrinsic curvature, with $k=\operatorname{tr}(\boldsymbol{k})$. Then, the gravitational Hamiltonian $H$ is given by $[35,45]$

$$
\begin{aligned}
H[\boldsymbol{h}, \boldsymbol{\pi}]= & \int_{\Sigma} \mathrm{d}^{3} x(N C+N \cdot \boldsymbol{C}) \\
& +\oint_{\partial \Sigma} \mathrm{d}^{2} x\left(-2 N \sqrt{\sigma} k+\frac{2}{N} N_{a} r_{b} \pi^{a b}\right),
\end{aligned}
$$

where, using $\pi=\operatorname{tr}(\boldsymbol{\pi})$ and $\mathcal{R}$ to denote the Ricci scalar on $\Sigma$,

$$
\begin{gathered}
C=-\sqrt{h} \mathcal{R}+\frac{1}{\sqrt{h}}\left(\pi: \pi-\frac{1}{2} \pi^{2}\right), \\
C_{a}=-2 D^{b} \pi_{a b}
\end{gathered}
$$

are the Hamiltonian and momentum constraints of GR, respectively. We can obtain from this Hamiltonian [Eq. (96)] the canonical equations of motion $\dot{h}_{a b}=$ $\left\{h_{a b}, H\right\}=\delta H / \delta \pi^{a b}$ and $\dot{\pi}_{a b}=\left\{\pi_{a b}, H\right\}=-\delta H / \delta h^{a b}$, in addition to the constraint equations $C=0=\boldsymbol{C}$.

Following the same procedure as before for a hypothetical entropy functional $S[\boldsymbol{h}, \boldsymbol{\pi}]$ and an equilibrium configuration $(\stackrel{\circ}{\boldsymbol{h}}, \boldsymbol{\pi})$ in phase space, we see that the Poisson bracket [Eq. (60)] in this case has the following form:

$$
\begin{aligned}
& \dot{S}=\int_{\Sigma} \mathrm{d}^{3} x \mathrm{~d}^{3} y \mathrm{~d}^{3} z\left\{\left[\frac{\delta^{2} H[\stackrel{\circ}{\boldsymbol{h}, \stackrel{\circ}{\boldsymbol{\pi}}]}}{\delta h^{c d}(y) \delta \stackrel{\circ}{\pi}^{a b}(x)} \frac{\delta^{2} S[\stackrel{\circ}{\boldsymbol{h}, ~ \stackrel{\circ}{\pi}]}}{\delta \stackrel{\circ}{h}_{e f}(z) \delta \stackrel{\circ}{h}_{a b}(x)}-\frac{\delta^{2} H[\stackrel{\circ}{\boldsymbol{h}, \stackrel{\circ}{\boldsymbol{\pi}}]}}{\delta \grave{\circ}^{c d}(y) \delta \stackrel{\circ}{h}^{a b}(x)} \frac{\delta^{2} S[\stackrel{\circ}{\boldsymbol{h}, \stackrel{\circ}{\boldsymbol{\pi}}]}}{\delta \stackrel{\circ}{h}_{e f}(z) \delta \stackrel{\circ}{\pi}_{a b}(x)}\right] \Delta h^{c d}(y) \Delta h_{e f}(z)\right.
\end{aligned}
$$

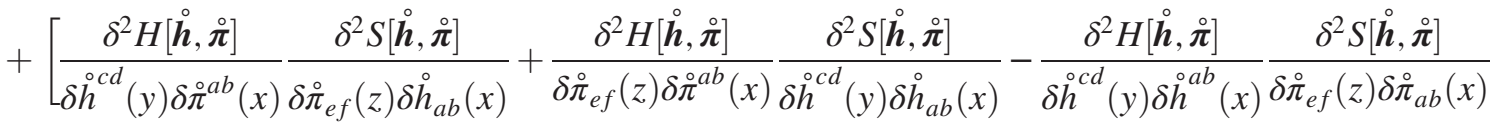

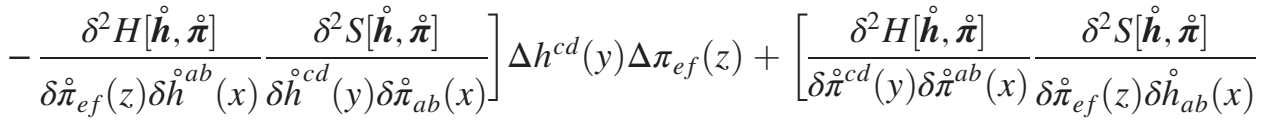

$$
\begin{aligned}
& \left.\left.-\frac{\delta^{2} H[\stackrel{\circ}{\boldsymbol{h}}, \stackrel{\circ}{\boldsymbol{\pi}}]}{\delta \stackrel{\circ}{\pi}^{c d}(y) \delta \stackrel{\circ}{h}^{a b}(x)} \frac{\delta^{2} S[\stackrel{\circ}{\boldsymbol{h}, \stackrel{\circ}{\boldsymbol{\pi}}]}}{\delta \stackrel{\circ}{\pi f}_{e f}(z) \delta \stackrel{\circ}{\pi}_{a b}(x)}\right] \Delta \pi^{c d}(y) \Delta \pi_{e f}(z)\right\}+\mathcal{O}\left(\Delta^{3}\right) .
\end{aligned}
$$


The difference with the previous cases is that here, in general, none of the second derivatives of the Hamiltonian vanishes, and, crucially, they do not have a definite sign. For example, let us compute the second derivative of $H$ with respect to the canonical momentum:

$$
\begin{aligned}
\frac{\delta^{2} H[\stackrel{\circ}{\boldsymbol{h}}, \stackrel{\circ}{\boldsymbol{\pi}}]}{\delta \stackrel{\circ}{\pi}_{c d}(y) \delta \stackrel{\circ}{\pi}^{a b}(x)}= & \frac{2 \stackrel{\circ}{N}(x)}{\sqrt{\stackrel{\circ}{h}(x)}}\left(\delta^{c}{ }_{(a} \delta^{d}{ }_{b)}-\frac{1}{2} \stackrel{\circ}{h}_{a b}(x) \delta^{c d}\right) \\
& \times \delta(x-y) .
\end{aligned}
$$

In CM or the examples of field theories in curved spacetime we have considered, the second derivative of $H$ with respect to the momentum had a definite sign (by virtue of its association with the positivity of kinetic-type terms). In this case, however, this second derivative [Eq. (100)] is neither always positive nor always negative. Thus, an argument similar to the previous proofs cannot work here: the gravitational Hamiltonian [Eq. (96)] is of such a nature that its concavity in phase space components (as is, for example, its concavity in the canonical momentum components [Eq. (100)]) is not independent of the phase space variables themselves and cannot be ascribed a definite (positive or negative) sign. And so, a contradiction cannot arise with the Poisson bracket of a phase space functional (such as the gravitational entropy) being nonzero (and, in particular, positive).

\section{Topological approach}

As discussed in Sec. IIIC, the topological proofs of Olsen for the nonexistence of entropy production in CM rely crucially on the assumption that the phase space $\mathscr{P}$ is compact. In such a situation, a system has a finite measure of phase space $\mu(\mathscr{P})$ available to explore, and there cannot exist a function which continually increases along orbits.

By contrast, in GR, it is believed that the (reduced) phase space $\mathscr{S}$ is generically noncompact [24]. That is to say, the measure $\mu(\mathscr{S})=\left.\int_{\mathscr{S}} \Omega\right|_{\mathscr{S}}$ in general diverges, where $\left.\Omega\right|_{\mathscr{S}}$ is (using the notation of Sec. II) the pullback of the symplectic form [Eq. (95)] to $\mathscr{S}$. This means that the same methods of proof as in CM (Sec. III C) cannot be applied.

The connection between a (monotonically increasing) entropy function in GR and the divergence of its (reduced) phase space measure warrants some discussion. The latter, it may be noted, is arguably not completely inevitable. In other words, one may well imagine a space of admissible solutions to the Einstein equations (or equivalently, the canonical gravitational equations) of which the effective degrees of freedom are such that they form a finite-measure phase space. Dynamically trivial examples of this might be SD black holes. Thus, the assertion that $\mu(\mathscr{S})$ diverges hinges on the nature of the degrees of freedom believed to be available in the spacetimes under consideration. However, it has been explicitly shown [24] that, even in very basic dynamically nontrivial situations, such as simple cosmological spacetimes, $\mu(\mathscr{S})$ does indeed diverge. In fact, the proof found in Ref. [24] is carried out for compact Cauchy surfaces, and the conclusion is therefore in concordance with the no-return theorem $[25,26]$ which also assumes compact Cauchy surfaces. In the following section, we will show that this happens for perturbed SD spacetimes as well (where the Cauchy surface is noncompact).

The generic divergence of $\mu(\mathscr{S})$ entails that a gravitational system has an unbounded region of phase space available to explore. In other words, it is not confined to a finite region where it would have to eventually return to a configuration from which it started (which would make a monotonically increasing entropy function impossible).

It is moreover worth remarking that this situation creates nontrivial problems for a statistical (i.e. probability-based) general-relativistic definition of entropy, $S: \mathscr{T} \rightarrow \mathbb{R}$ (as described in Secs. I and II)—which, indeed, one may also ultimately desire to work with and relate to the mechanical meaning of entropy mainly discussed in this paper. Naively, one might think of defining such a statistical entropy function as something along the lines of $S=-\sum_{X} P(X) \ln P(X)$, where $X$ denotes a physical property of interest and $P(X)$ its probability. In turn, the latter might be understood as the relative size of the phase space region $\mathscr{S}_{X} \subset \mathscr{S}$ possessing the property $X$, i.e. $P(X)=\mu\left(\mathscr{S}_{X}\right) / \mu(\mathscr{S})$. In this case, we either have [24] $P(X)=0$ if $\mu\left(\mathscr{S}_{X}\right)$ is finite, $P(X)=1$ if $\mu\left(\mathscr{S} \backslash \mathscr{S}_{X}\right)$ is finite, or $P(X)$ is ill defined otherwise. Ostensibly, one would need to invoke a regularization procedure in order to obtain finite probabilities (in general) according to this. However, different regularization procedures that have been applied (mainly in the context of cosmology) have proven to yield widely different results depending on the method of the procedure being used [24]. Alternatively, a statistical general-relativistic definition of $S$ in terms of a probability density $\rho: \mathscr{S} \times \mathscr{T} \rightarrow[0,1]$ (similarly to $\mathrm{CM}$ ) as $S=-\left.\int_{\mathscr{S}} \Omega\right|_{\mathscr{S}} \rho \ln \rho$ would likewise face divergence issues. Therefore, any future attempt to define gravitational entropy in such a context will have to either devise an unambiguous and well-defined regularization procedure (for obtaining finite probabilities) or implement a welljustified cutoff of the (reduced) phase space measure.

We now turn to discussing these issues in a context where we expect an intuitive illustration of gravitational entropy production - the two-body problem.

\section{ENTROPY IN THE GRAVITATIONAL TWO-BODY PROBLEM}

One of the most elementary situations in GR in which we expect the manifestation of a phenomenon such as entropy production is the gravitational two-body problem.

In CM, the two-body (or Kepler) problem manifestly involves no increase in the entropy of a system. The 
perturbative approach, as discussed in Sec. III B 3, involves assumptions on the nature of the Hamiltonian which preclude any conclusions from it in this regard. However, the topological approach, elaborated in Sec. III C, is applicable: assuming that Keplerian orbits are bounded, the configuration space $\mathscr{Q}$ can be considered to be compact, and therefore the phase space $\mathscr{P}$ obtained from it (involving finite conjugate momenta) is compact as well. Concordant with the topological proofs, then, we will have no entropy production in such a situation. The case of the $N$-body problem, however, is, as alluded to earlier, not the same; neither the assumptions of the perturbative approach nor of the topological approach (specifically, a compact phase space) are applicable, and it has been shown that a monotonically increasing function on phase space does in fact exist [14,15], and hence so does a gravitational arrow of time (and entropy production) associated with it.

In GR, we know the two-body problem involves energy loss and therefore should implicate an associated production of entropy. The no-return theorem $[25,26]$ is inapplicable here because this problem does not involve a compact Cauchy surface. The perturbative approach here fails to disprove the second law (as discussed in Sec. IV B 3), and we will now show that so, too, does the topological approach.

The two-body problem in GR where one small body orbits a much larger body of mass $M$ can be modeled in the context of perturbations to the SD metric,

$$
g_{a b} \mathrm{~d} x^{a} \mathrm{~d} x^{b}=-v(r) \mathrm{d} t^{2}+\frac{\mathrm{d} r^{2}}{v(r)}+r^{2} \sigma_{a b} \mathrm{~d} x^{a} \mathrm{~d} x^{b}
$$

where $v(r)=1-2 M / r$ and $\sigma=\operatorname{diag}\left(0,0,1, \sin ^{2} \theta\right)$ is the metric of the 2 -sphere $\mathbb{S}^{2}$. According to standard black hole perturbation theory (see, for example, Refs. [46-48]), it is possible to choose a gauge so that the polar and axial parts of perturbations to this metric are encoded in a single gauge-invariant variable each. In particular, they are given, respectively, by

$$
\Phi_{( \pm)}=\frac{1}{r} \sum_{l=0}^{\infty} \sum_{m=-l}^{l} Y^{l m}(\theta, \phi) \Psi_{( \pm)}^{l m}(t, r),
$$

where $Y^{l m}$ are spherical harmonics and $\Psi_{( \pm)}^{l m}$ are called, respectively, the Zerilli and Regge-Wheeler master functions, which satisfy known wavelike equations and from which the perturbations to $g$ can be reconstructed. In Ref. [34], the symplectic form of the reduced phase space $\mathscr{S}$ for such spacetimes is computed. Without entering into the details of the computation, we simply state the result,

$$
\left.\Omega\right|_{\mathscr{S}}=\sum_{\varsigma= \pm} \int_{\Sigma} \mathrm{d}^{3} x \delta \Upsilon_{(\varsigma)} \wedge \mathbb{D} \delta \Phi_{(\varsigma)}
$$

where $\Upsilon_{( \pm)}=\left[r^{2} \sin \theta / v(r)\right] \dot{\Phi}_{( \pm)}$, and $\mathbb{D}=\Delta_{\sigma}^{-1}\left(\Delta_{\sigma}+2\right)^{-1}$, where $\Delta_{\sigma}$ is the Laplace operator on $\mathbb{S}^{2}$.

The work [34] where this symplectic form [Eq. (103)] was derived simply uses it to define and formulate conservation laws for energy and angular momentum in perturbed SD spacetimes. It does not, however, address the question of the total measure of $\mathscr{S}$. We will now show that the (reduced) phase space measure $\mu(\mathscr{S})=\left.\int_{\mathscr{S}} \Omega\right|_{\mathscr{S}}$ for such spacetimes in fact diverges, preventing any argument based on phase space compactness for the nonexistence of entropy production.

Inserting the definitions of the different variables and suppressing for the moment the coordinate dependence of the spherical harmonics and master functions, we have

$$
\begin{aligned}
\mu(\mathscr{S})= & \sum_{\varsigma= \pm} \int_{\mathscr{S}} \int_{\Sigma} \mathrm{d}^{3} x \delta \Upsilon_{(\varsigma)} \wedge \mathbb{D} \delta \Phi_{(\varsigma)} \\
= & \sum_{\varsigma= \pm} \int_{\mathscr{S}} \int_{\Sigma} \mathrm{d}^{3} x \delta\left(\frac{r^{2} \sin \theta}{v(r)} \dot{\Phi}_{(\varsigma)}\right) \wedge \mathbb{D} \delta \Phi_{(\varsigma)} \\
= & \sum_{\varsigma= \pm} \int_{\mathscr{S}} \int_{\Sigma} \mathrm{d}^{3} x \delta\left(\frac{r \sin \theta}{v(r)} \sum_{l, m} Y^{l m} \dot{\Psi}_{(\varsigma)}^{l m}\right) \\
& \wedge \mathbb{D} \delta\left(\frac{1}{r} \sum_{l^{\prime}, m^{\prime}} Y^{l^{\prime} m^{\prime}} \Psi_{(\varsigma)}^{l^{\prime} m^{\prime}}\right) .
\end{aligned}
$$

Now, using the fact that the functional exterior derivative acts only on the master functions and the operator $\mathbb{D}$ acts only on the spherical harmonics, we can write this as

$$
\begin{aligned}
\mu(\mathscr{S})= & \sum_{\varsigma= \pm} \int_{\mathscr{S}} \int_{\Sigma} \mathrm{d}^{3} x\left(\frac{r \sin \theta}{v(r)} \sum_{l, m} Y^{l m} \delta \dot{\Psi}_{(\varsigma)}^{l m}\right) \\
& \wedge\left(\frac{1}{r} \sum_{l^{\prime}, m^{\prime}}\left(\mathbb{D} Y^{l^{\prime} m^{\prime}}\right) \delta \Psi_{(\varsigma)}^{l^{\prime} m^{\prime}}\right) \\
= & \sum_{\varsigma= \pm} \sum_{l, l^{\prime}, m, m^{\prime}} \int_{\mathscr{S}} \int_{\Sigma} \mathrm{d}^{3} x\left(\frac{r \sin \theta}{v(r)} Y^{l m} \frac{1}{r} \mathbb{D} Y^{l^{\prime} m^{\prime}}\right) \\
& \times \delta \dot{\Psi}_{(\varsigma)}^{l m} \wedge \delta \Psi_{(\varsigma)}^{l^{\prime} m^{\prime}} .
\end{aligned}
$$

Writing the Cauchy surface integral in terms of coordinates and collecting terms,

$$
\mu(\mathscr{S})=\sum_{\varsigma= \pm} \sum_{l, l^{\prime}, m, m^{\prime}} \int_{\mathscr{S}} \int_{2 M}^{\infty} \mathrm{d} r \int_{\mathbb{S}^{2}} \mathrm{~d} \theta \mathrm{d} \phi \frac{1}{v(r)}\left[(\sin \theta) Y^{l m} \mathbb{D} Y^{l^{l^{\prime} m^{\prime}}}\right] \delta \dot{\Psi}_{(\varsigma)}^{l m} \wedge \delta \Psi_{(\varsigma)}^{l^{\prime{ }^{\prime} m^{\prime}}}
$$




$$
\begin{aligned}
& =\sum_{\varsigma= \pm} \sum_{l, l^{\prime}, m, m^{\prime}} \int_{\mathscr{S}}\left[\int_{\mathbb{S}^{2}} \mathrm{~d} \theta \mathrm{d} \phi(\sin \theta) Y^{l m} \mathbb{D} Y^{l^{\prime} m^{\prime}}\right] \int_{2 M}^{\infty} \frac{\mathrm{d} r}{v(r)} \delta \dot{\Psi}_{(\varsigma)}^{l m} \wedge \delta \Psi_{(\varsigma)}^{l^{\prime} m^{\prime}} \\
& =\sum_{\varsigma= \pm} \sum_{l, l^{\prime}, m, m^{\prime}} A^{l l^{\prime} m m^{\prime}} \int_{\mathscr{S}} \int_{2 M}^{\infty} \frac{\mathrm{d} r}{v(r)} \delta \dot{\Psi}_{(\varsigma)}^{l m} \wedge \delta \Psi_{(\varsigma)}^{l^{\prime} m^{\prime}}
\end{aligned}
$$

where $A^{l l^{\prime} m m^{\prime}}=\int_{\mathbb{S}^{2}} \mathrm{~d} \theta \mathrm{d} \phi(\sin \theta) Y^{l m} \mathbb{D} Y^{l^{\prime} m^{\prime}}$ is a finite integral involving only the spherical harmonics. Restoring the arguments of the master functions - and recalling that, for any function $f(t, r)$, the meaning of $\delta f(t, r)$ is simply that of a 1form on the phase space at $(t, r)$ in spacetime-we can write from the above [Eq. (111)]

$$
\begin{aligned}
\mu(\mathscr{S}) & =\sum_{\varsigma= \pm} \sum_{l, l^{\prime}, m, m^{\prime}} A^{l l^{\prime} m m^{\prime}} \int_{\mathscr{S}} \int_{2 M}^{\infty} \frac{\mathrm{d} r}{v(r)}\left[\delta \dot{\Psi}_{(\varsigma)}^{l m}(t, r) \wedge \delta \Psi_{(\varsigma)}^{l^{\prime} m^{\prime}}(t, r)\right] \\
& \geq \sum_{\varsigma= \pm l, l^{\prime}, m, m^{\prime}} A^{l l^{\prime} m m^{\prime}} \int_{\mathscr{S}} \int_{2 M}^{\infty} \frac{\mathrm{d} r}{v(r)}\left[\min _{\bar{r} \in[2 M, \infty)} \delta \dot{\Psi}_{(\varsigma)}^{l m}(t, \bar{r}) \wedge \delta \Psi_{(\varsigma)}^{l^{\prime} m^{\prime}}(t, \bar{r})\right] \\
& =\sum_{\varsigma= \pm l, l^{\prime}, m, m^{\prime}} A^{l l^{\prime} m m^{\prime}}\left[\int_{\mathscr{S}} \min _{\bar{r} \in[2 M, \infty)} \delta \dot{\Psi}_{(\varsigma)}^{l m}(t, \bar{r}) \wedge \delta \Psi_{(\varsigma)}^{l^{\prime} m^{\prime}}(t, \bar{r})\right] \int_{2 M}^{\infty} \frac{\mathrm{d} r}{v(r)} \\
& =\left\{\sum_{\zeta= \pm} \sum_{l, l^{\prime}, m, m^{\prime}} A^{l l^{\prime} m m^{\prime}}\left[\min _{\bar{r} \in[2 M, \infty)} \int_{\mathscr{S}} \delta \dot{\Psi}_{(\varsigma)}^{l m}(t, \bar{r}) \wedge \delta \Psi_{(\varsigma)}^{l^{\prime} m^{\prime}}(t, \bar{r})\right]\right\}\left[\int_{2 M}^{\infty} \frac{\mathrm{d} r}{v(r)}\right] .
\end{aligned}
$$

The phase space integral $\int_{\mathscr{S}} \delta \dot{\Psi}_{(\varsigma)}^{l m}(t, \bar{r}) \wedge \delta \Psi_{(\varsigma)}^{l^{\prime \prime} m^{\prime}}(t, \bar{r})$ is finite but nonzero even when minimized over $\bar{r}$ because for any nontrivial solutions of the master functions there will be no point in spacetime where they will always be vanishing (for all time). Thus (assuming that the $l, l^{\prime}, m, m^{\prime}$ sums are convergent), everything in the curly bracket in the last line above [Eq. (115)] is nonzero but finite. However, it multiplies $\int_{2 M}^{\infty} \mathrm{d} r / v(r)$, which diverges (at both integration limits). Hence, $\mu(\mathscr{S})$ diverges for such spacetimes.

We can make a few remarks. First, one might be concerned in the above argument, specifically in the last line [Eq. (115)], about what might happen in the asymptotic limit of the phase space integral; in other words, it maybe the case (i) that $\min _{\bar{r} \in[2 M, \infty)} \int_{\mathscr{S}} \delta \dot{\Psi}_{(\varsigma)}^{l m}(t, \bar{r}) \wedge \delta \Psi_{(\varsigma)}^{l^{\prime} m^{\prime}}(t, \bar{r})$ could turn out to be $\lim _{r \rightarrow \infty} \int_{\mathscr{S}} \delta \dot{\Psi}_{(\varsigma)}^{l m}(t, r) \wedge \delta \Psi_{(\varsigma)}^{l^{\prime} m^{\prime}}(t, r)$, and, if so, one might naively worry (ii) that the latter vanishes due to asymptotic decay properties of the master functions. This will actually not happen. To see why, suppose (i) is true. The master functions must obey outgoing boundary conditions at spatial infinity, i.e. $0=\left[\partial_{t}+v(r) \partial_{r}\right] \Psi_{(\varsigma)}^{l m}$ as $r \rightarrow \infty$. Hence, we have

$$
\begin{aligned}
\lim _{r \rightarrow \infty} \int_{\mathscr{S}} \delta \dot{\Psi}_{(\varsigma)}^{l m} \wedge \delta \Psi_{(\varsigma)}^{l^{\prime} m^{\prime}} & =\lim _{r \rightarrow \infty} \int_{\mathscr{S}} \delta\left(-v \partial_{r} \Psi_{(\varsigma)}^{l m}\right) \wedge \delta \Psi_{(\varsigma)}^{l^{\prime} m^{\prime}} \\
& =-\int_{\mathscr{S}} \lim _{r \rightarrow \infty} \delta\left(\partial_{r} \Psi_{(\varsigma)}^{l m}\right) \wedge \delta \Psi_{(\varsigma)}^{l^{\prime} m^{\prime}},
\end{aligned}
$$

which is nonzero, because the vanishing of the master functions and their radial partials at spatial infinity for all time corresponds only to trivial solutions. Therefore, we have that $\min _{\bar{r} \in[2 M, \infty)} \int_{\mathscr{S}} \delta \dot{\Psi}_{(\varsigma)}^{l m}(t, \bar{r}) \wedge$ $\delta \Psi_{(\varsigma)}^{l^{\prime} m^{\prime}}(t, \bar{r})$ is always nonzero for nontrivial solutions.

Second, if the two-body system in this framework is an extreme-mass-ratio inspiral [29], i.e. the mass of the orbiting body, or "particle," is orders of magnitude smaller than that of the larger one, and the former is modeled using a stress-energy-momentum tensor with support only on its worldline, then it is known that $\Psi_{( \pm)}^{l m}(t, r)$ has a discontinuity at the particle location, and thus $\dot{\Psi}_{( \pm)}^{l m}(t, r)$ has a divergence there. Hence, the integral over $\mathscr{S}$ even before our inequality above [Eq. (112)] is already divergent due to the divergence of $\dot{\Psi}_{( \pm)}^{l m}(t, r)$ in the integrand. However, given that such an approach to describing these systems (i.e. having a stress-energy-momentum tensor of the particle with a delta distribution) is only an idealization, we regard the conclusion that $\mu(\mathscr{S})$ diverges as more convincing based on our earlier argument, which is valid in general-that is, even for possible descriptions of the smaller body that may be more realistic than that using delta distributions.

\section{CONCLUSIONS}

We have proven that there does not exist a monotonically increasing function of phase space-which may be 
identified as (what we have referred to as a mechanical notion of) entropy-in classical mechanics with $N$ degrees of freedom for certain classes of Hamiltonians, as well as in some (classical) matter field theories in curved (nondynamical) spacetime, viz. for standard scalar and electromagnetic fields. To do this, we have followed the procedure for the proof sketched by Poincaré [22] (what we have dubbed the perturbative approach), and we have here carried it out in full rigour for classical mechanics and extended it via similar techniques to field theories. What is noteworthy about this perturbative proof - counter (to our knowledge) to all other well-known proofs for the nonexistence of entropy (in the mechanical sense) in classical canonical theories-is that it assumes nothing about the topology of the phase space; in other words, the phase space can be noncompact. Essentially, it relies only on curvature properties (in phase space) of the Hamiltonian of the canonical theory being considered. We have explicated these properties in the case of classical mechanics and have assumed standard ones for the particular (curved spacetime) matter field theories we have investigated. It would be of interest for future work to determine, in the case of the former whether they can be made less restrictive (than what we have required for our proof, which thus omits some classes of Hamiltonians of interest such as that for the gravitational two-body problem) and in the case of the latter whether they can be generalized or extended to broader classes of field theories. Indeed, it would be in general an interesting question to determine not only the necessary but also-if possible - the sufficient conditions that a Hamiltonian of a generic canonical theory needs to satisfy in order for this theorem to be applicable, i.e. in order to preclude mechanical entropy production. We have seen that it is precisely the curvature properties of the vacuum Hamiltonian of general relativity that prevent this method of proof from being extended thereto, where in fact one does expect (some version of) the second law of thermodynamics to hold.

Topological properties of the phase space can also entail the nonexistence of mechanical entropy, as per the more standard and already well-understood proofs in classical mechanics where the phase space is assumed to be compact $[13,23]$. However, even for nongravitational canonical theories, this assumption might be too restrictive, and for general relativity, it is believed that in general it is not the case. This renders any of these topological proofs inapplicable in the case of the latter, and moreover it also significantly complicates any attempt to formulate a sensible "statistical" notion of (gravitational) entropy due to the concordant problems in working with finite probabilities (of phase space properties). These must ultimately be overcome (via some regularization procedure or cutoff argument) for establishing a connection between a statistical and mechanical entropy in general relativity. While we still lack any consensus on how to define the latter, it may be hoped that in the future the generic validity of a (general-relativistic) second law may be demonstrated on the basis of (perhaps curvature related) properties of the gravitational Hamiltonian-which in turn may enter into a statistical mechanics type definition of gravitational entropy in terms of some suitably defined partition function. In this regard, older work based on field-theoretic approaches [49] and more recent developments such as proposals to relate entropy with a Noether charge (specifically, the Noether invariant associated with an infinitesimal time translation) in classical mechanics [50] may provide fruitful hints.

A clear situation in which we anticipate entropy production in general relativity, unlike in classical mechanics, is the gravitational two-body problem. For the latter, as we have discussed, the $N$-body problem actually does also exhibit features of entropy production. We have shown here explicitly that the phase space of perturbed SchwarzschildDroste spacetimes is noncompact (even without the assumption of self-force). This means that the topological proofs are here inapplicable (but also, on the other hand, so is the no-return theorem for compact Cauchy surfaces, which by itself cannot be used in this case to understand the nonrecurrence of phase space orbits). It is hoped that, once a generally agreed upon definition of gravitational entropy is established, one would not only be able to use it to compute the entropy of two-body systems but also to demonstrate that it should obey the second law (i.e. that it should be monotonically increasing in time). In the long run, an interesting problem to investigate is whether an entropy change, once defined and associated to motion in a Lagrangian formulation, could determine the trajectory of a massive and radiating body, moving in a gravitational field.

\section{ACKNOWLEDGMENTS}

M. O. would like to thank Richard J. Epp (Waterloo) and César Ramírez Ibañez (Waterloo) for useful discussions. M. O. has received financial support through Eiffel Bourse d'Excellence Grant No. 840856D by Campus France and through the Natural Sciences and Engineering Research Council of Canada. M. O., L. B., and A.D. A. M. S. acknowledge LISA CNES funding. C. F. S. acknowledges support from Contracts No. ESP2013-47637-P and No. ESP2015-67234-P (Spanish Ministry of Economy and Competitivity of Spain, MINECO).

\section{APPENDIX: SOME DETAILS ON CONSTRAINED HAMILTONIAN SYSTEMS}

In this Appendix, we elaborate on some technical details omitted from Secs. II and III.

In $\mathscr{P}$, the symplectic form $\boldsymbol{\omega}$ is used to define the Hamiltonian vector field $\boldsymbol{X}_{F}$ of any phase space function $F: \mathscr{P} \rightarrow \mathbb{R}$ via $l_{\boldsymbol{X}_{F}} \boldsymbol{\omega}=-\mathrm{d} F$, where $\iota$ is the interior product 


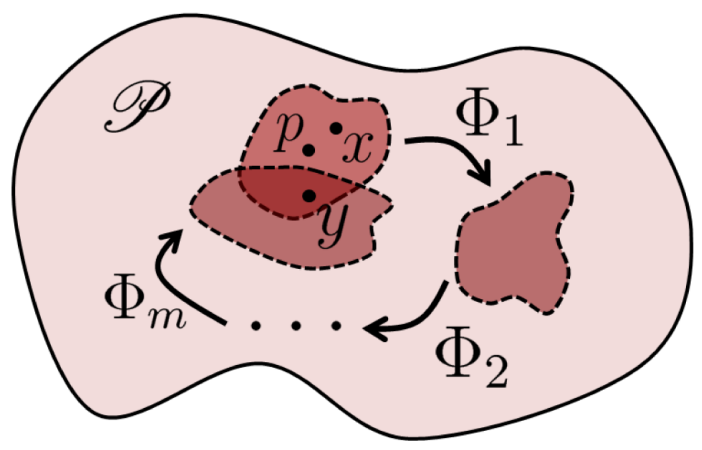

FIG. 3. The idea of the proof for the Poincare recurrence theorem.

and $\mathrm{d}$ is the exterior derivative on $\mathscr{P}$. The Hamiltonian flow (generated by $\boldsymbol{X}_{H}$ ) is determined via $\mathrm{d} \Phi_{t} / \mathrm{d} t=\boldsymbol{X}_{H} \circ \Phi_{t}$.

Using this, we can easily offer a proof, shown pictorially in Fig. 3, for the recurrence theorem (see Sec. 16 of Ref. [18]): assume $\mathscr{P}$ is compact and $\Phi_{t}(\mathscr{P})=\mathscr{P}$. Let $\mathscr{U} \subset \mathscr{P}$ be the neighborhood of any point $p \in \mathscr{P}$, and consider the sequence of images $\left\{\Phi_{n}(\mathscr{U})\right\}_{n=0}^{\infty}$. Each $\Phi_{n}(\mathscr{U})$ has the same measure $\int_{\Phi_{n}(\mathscr{U})} \Omega$ (because of Liouville's theorem), so if they never intersected, $\mathscr{P}$ would have infinite measure. Therefore, there exist $k, l$ with $k>l$ such that $\Phi_{k}(\mathscr{U}) \cap \Phi_{l}(\mathscr{U}) \neq \emptyset$, implying $\Phi_{m}(\mathscr{U}) \cap \mathscr{U} \neq$ $\emptyset$ where $m=k-l$. For any $y \in \Phi_{m}(\mathscr{U}) \cap \mathscr{U}$, there exists an $x \in \mathscr{U}$ such that $y=\Phi_{m}(x)$. Thus, any point returns arbitrarily close to the initial conditions in a compact and invariant phase space.

Let us now describe how one must deal with constraints in a Hamiltonian system, i.e. how to go from $(\mathscr{P}, \boldsymbol{\omega})$ to $\left(\mathscr{S},\left.\omega\right|_{\mathscr{S}}\right)$ (in the notation already anticipated in Sec. II). Let $C_{I}: \mathscr{P} \rightarrow \mathbb{R}$ denote a set of phase space functions indexed by $I$, and suppose the system is subject to the constraint equations $C_{I}=0$. Then, one is only interested in the constraint surface of the phase space, $\mathscr{C} \subset \mathscr{P}$, where the constraint equations are satisfied. Let $\left.\boldsymbol{\omega}\right|_{\mathscr{C}}$ denote the pullback of the phase space symplectic form $\boldsymbol{\omega}$, onto the constraint surface $\mathscr{C}$. Depending on the nature of the constraints $C_{I},\left.\boldsymbol{\omega}\right|_{\mathscr{C}}$ may be degenerate and therefore not a symplectic form on $\mathscr{C}$. In particular, if any one of the constraints is first class-meaning that its Hamiltonian vector field is everywhere tangent to $\mathscr{C}$-then $\left.\boldsymbol{\omega}\right|_{\mathscr{C}}$ is degenerate and hence cannot furnish a symplectic structure. Only if all constraints are second class-i.e. their Hamiltonian vector fields are nowhere tangent to $\mathscr{C}$-will one obtain a pullback which is itself a symplectic form.

In many situations of interest for classical field theories (as is the case, for example, in both Maxwellian EM and GR), the constraints are first class, and the degeneracy directions of $\left.\omega\right|_{\mathscr{C}}$ correspond precisely to pure gauge variations of the fields. In other words, the kernel of $\left.\boldsymbol{\omega}\right|_{\mathscr{C}}$ comprises the vector fields of which the flow in phase space represents gauge transformations [of the $\mathbb{U}(1)$ gauge symmetry in Maxwellian EM and of the diffeomorphism invariance of GR]. In principle, a symplectic form could be obtained if one factors out these vector fields, by identifying all points on the orbits of their flow. Thus, one could work with a factor space $\tilde{\mathscr{P}} \subset \mathscr{C}$ which is simply the space of gauge orbits in $\mathscr{C}$ and which is symplectic.

However, depending on the desired aim of implementing the canonical construction, taking such an approach can be problematic. This is because in any theory which is diffeomorphism invariant (such as GR) "time evolution" is effected via spacetime diffeomorphisms, and so moving to the space of gauge orbits essentially renders the dynamics nonexistent; it become entirely trivial, because it is essentially factored out of $\tilde{\mathscr{P}}$, leaving one with no more sense of "motion through phase space."

There exist two possible solutions for ameliorating this difficulty - that is, for obtaining a symplectic structure out of $\left.\boldsymbol{\omega}\right|_{\mathscr{C}}$ which does still preserve a nontrivial notion of time evolution: (a) Instead of passing to the space of gauge orbits, one may instead choose a representative of each gauge orbit [24]. The idea is that one can find a surface $\mathscr{S} \subset \mathscr{C}$ such that each gauge orbit in $\mathscr{C}$ intersects $\mathscr{S}$ once and only once. (In fact, sometimes a family of such surfaces that work in localized regions of $\mathscr{C}$ is needed, but we keep our discussion here simplified.) The choice of $\mathscr{S}$ is not unique, and so taking a different surface $\mathscr{S}^{\prime}$ basically amounts to a change of descriptioncorresponding to time evolution (i.e. change of representative Cauchy surface in spacetime) along with the associated spatial diffeomorphisms. The technicalities of this procedure are elaborated in Ref. [24], but the point is that the subspace $\mathscr{S}$ of the constraint surface $\mathscr{C}$ resulting from such a construction is symplectic, whence one can work with the symplectic form $\left.\omega\right|_{\mathscr{S}}$ obtained by pulling back $\left.\boldsymbol{\omega}\right|_{\mathscr{C}}$ to $\mathscr{S}$. (b) A specific choice of gauge may be imposed, such that the combination of the constraints $C_{I}$ together with the gauge-fixing conditions becomes second class [35]. One can thus obtain a symplectic structure on the subspace of the constraint surface $\mathscr{S} \subset \mathscr{C}$ where the (appropriately chosen) gauge-fixing conditions are satisfied and where one will thus have a symplectic form $\left.\omega\right|_{\mathscr{S} \text {. }}$

In order to keep our discussion general, we use the notation $\left(\mathscr{S},\left.\boldsymbol{\omega}\right|_{\mathscr{S}}\right)$ to refer to the "reduced phase space" irrespective of whether procedure (a) or (b) is used to define it. 
[1] A. Einstein, Autobiographical Notes (Open Court, Chicago, 1999).

[2] L. Sklar, Physics and Chance: Philosophical Issues in the Foundations of Statistical Mechanics (Cambridge University Press, Cambridge, England, 1995).

[3] P. C. W. Davies, The Physics of Time Asymmetry (University of California, Berkeley and Los Angeles, 1977).

[4] H. R. Brown, W. Myrvold, and J. Uffink, Boltzmann's Htheorem, its discontents, and the birth of statistical mechanics, Stud. Hist. Philos. Sci. B Stud. Hist. Philos. Mod. Phys. 40, 174 (2009).

[5] C. Rovelli, General relativistic statistical mechanics, Phys. Rev. D 87, 084055 (2013).

[6] E. P. Verlinde, On the origin of gravity and the laws of Newton, J. High Energy Phys. 04 (2010) 029.

[7] M. H. P. M. van Putten, Entropic force in black hole binaries and its Newtonian limits, Phys. Rev. D 85, 064046 (2012).

[8] S. M. Carroll and G. N. Remmen, What is the entropy in entropic gravity?, Phys. Rev. D 93, 124052 (2016).

[9] L. Freidel, Gravitational energy, local holography and nonequilibrium thermodynamics, Classical Quantum Gravity 32, 055005 (2015).

[10] L. Freidel and Y. Yokokura, Non-equilibrium thermodynamics of gravitational screens, Classical Quantum Gravity 32, 215002 (2015).

[11] L. Boltzmann, Weitere studien über das wärmegleichgewicht unter gasmolekülen, Sitzungsber. der Akad. der Wiss. Wien II 66, 275 (1872).

[12] J. J. Loschmidt, Über den zustand des wärmegleichgewichtes eines systems von körpern mit rücksicht auf die schwerkraft, Sitzungsber. Akad. Wiss. Wien Math. Naturwiss. K1. Abt. 1 73, 128 (1876).

[13] H. Poincaré, Sur le problème des trois corps et les équations de la dynamique, Acta Math. 13, 1 (1890).

[14] J. Barbour, T. Koslowski, and F. Mercati, A Gravitational Origin of the Arrows of Time, arXiv:1310.5167.

[15] J. Barbour, T. Koslowski, and F. Mercati, Identification of a Gravitational Arrow of Time, Phys. Rev. Lett. 113, 181101 (2014).

[16] V. Sahni, Y. Shtanov, and A. Toporensky, Arrow of time in dissipationless cosmology, Classical Quantum Gravity 32, 182001 (2015).

[17] V. Sahni and A. Toporensky, Cosmological hysteresis and the cyclic universe, Phys. Rev. D 85, 123542 (2012).

[18] V. I. Arnold, Mathematical Methods of Classical Mechanics, 2nd ed. (Springer, New York, 1997).

[19] L. Barreira, Poincaré recurrence: old and new, edited by J.-C. Zambrini, XIVth International Congress on Mathematical Physics, Lisbon, 2003 (World Scientific, Singapore, 2006), p. 415.

[20] C. Villani, H-Theorem and beyond: Boltzmann's entropy in today's mathematics, edited by G. Gallavotti, W. L. Reiter, and J. Yngvason, Boltzmann's Legacy, ESI Lectures in Mathematics and Physics, page 129. Eur. Math. Soc., 2008. Symposium held at the Erwin Schrödinger International Institute for Mathematical Physics, Vienna, 7-9 June, 2006.

[21] H.-T. Yau, Proceedings of the International Congress of Mathematicians, Hyderabad, India, 2010, edited by
R. Bhatia (Hindustan Book Agency, New Delhi, India, 2010), p. 87.

[22] H. Poincaré, Sur les tentatives d'explication mecanique des principes de la thermodynamique, C. R. Seances Acad. Sci. (Paris) 108, 550 (1889).

[23] E. T. Olsen, Classical mechanics and entropy, Found. Phys. Lett. 6, 327 (1993).

[24] J. S. Schiffrin and R. M. Wald, Measure and probability in cosmology, Phys. Rev. D 86, 023521 (2012).

[25] F. J. Tipler, General relativity, thermodynamics, and the Poincaré cycle, Nature (London) 280, 203 (1979).

[26] F. J. Tipler, Essays in General Relativity (Academic, New York, 1980), p. 21.

[27] R. P. A. C. Newman, Compact space-times and the no-return theorem, Gen. Relativ. Gravit. 18, 1181 (1986).

[28] B. P. Abbott et al., Observation of Gravitational Waves from a Binary Black Hole Merger, Phys. Rev. Lett. 116, 061102 (2016).

[29] L. Blanchet, A. Spallicci, and B. Whiting (editors), Mass and Motion in General Relativity. Fundamental Theories of Physics (Springer, New York, 2011), Vol. 162.

[30] J. Droste, Doctorate thesis, Rijksuniversiteit van Leiden, 1916.

[31] J. Droste, Het veld van een enkel centrum in Einstein's theorie der zwaartekracht, en de beweging van een stoffelijk punt in dat veld, Kon. Neder. Akad. van Weten. Amst. 25, 163 (1916) [Proc. Acad. Sc. Amsterdam 19, 197 (1917)].

[32] K. Schwarzschild, Über das gravitationsfeld eines massenpunktes nach der Einsteinschen theorie, Sitzungsber. Preuß. Akad. Wissenschaften Berlin, Phys.-Math. Kl., 189 (1916).

[33] T. Rothman, Editor's note: The field of a single centre in einstein's theory of gravitation, and the motion of a particle in that field, Gen. Relativ. Gravit. 34, 1541 (2002).

[34] J. Jezierski, Energy and angular momentum of the weak gravitational waves on the schwarzschild background quasilocal gauge-invariant formulation, Gen. Relativ. Gravit. 31, 1855 (1999).

[35] M. Bojowald, Canonical Gravity and Applications: Cosmology, Black Holes, and Quantum Gravity (Cambridge University Press, Cambridge, England, 2011).

[36] A. Hanson, T. Regge, and C. Teitelboim, Constrained Hamiltonian Systems (Accademia Nazionale dei Lincei, Rome, 1976).

[37] L. V. Abad, Principles of classical statistical mechanics: A perspective from the notion of complementarity, Ann. Phys. (Berlin) 327, 1682 (2012).

[38] H. Hofer and E. Zehnder, Symplectic Invariants and Hamiltonian Dynamics (Springer, New York, 2011).

[39] S. Suhr and K. Zehmisch, Linking and closed orbits, Abh. Math. Semin. Univ. Hambg. 86, 133 (2016).

[40] C. Crnković, Symplectic geometry and (super-)Poincaré algebra in geometrical theories, Nucl. Phys. B288, 419 (1987).

[41] C. Crnković and E. Witten, Three Hundred Years of Gravitation, edited by S. W. Hawking and W. Israel (Cambridge University Press, Cambridge, England, 1989), p. 676.

[42] R. M. Dreizler and E. Engel, Density Functional Theory (Springer, New York, 2011). 
[43] E. Poisson, A Relativist's Toolkit: The Mathematics of Black-Hole Mechanics (Cambridge University Press, Cambridge, England, 2007).

[44] C. Prescod-Weinstein and E. Bertschinger, An extension of the Faddeev-Jackiw technique to fields in curved spacetimes, Classical Quantum Gravity 32, 075011 (2015).

[45] R. M. Wald, General Relativity (University of Chicago, Chicago, 1984).

[46] S. Chandrasekhar, The Mathematical Theory of Black Holes (Clarendon, Oxford, 1998).
[47] V. Frolov and I. Novikov, Black Hole Physics: Basic Concepts and New Developments (Springer, New York, 1998).

[48] L. R. Price, Ph. D. thesis, University of Florida, 2007.

[49] G. Horwitz, Steepest descent path for the microcanonical ensemble-resolution of an ambiguity, Commun. Math. Phys. 89, 117 (1983).

[50] S. Sasa and Y. Yokokura, Thermodynamic Entropy as a Noether Invariant, Phys. Rev. Lett. 116, 140601 (2016). 